\title{
Delivery of chondroitinase by canine mucosal olfactory ensheathing cells alongside rehabilitation enhances recovery after spinal cord injury
}

Jon Prager ${ }^{a, b}$, Daisuke Ito ${ }^{c, d}$, Darren R. Carwardine ${ }^{a}$, Prince Jijuc ${ }^{c}$ Divya M. Charie, Nicolas Granger ${ }^{b}$, Liang-Fong Wong ${ }^{c *}$

a Bristol Veterinary School, University of Bristol, Bristol, UK

${ }^{\mathrm{b}}$ The Royal Veterinary College, University of London, Hatfield, UK

c Bristol Medical School, University of Bristol, Bristol, UK

${ }^{\mathrm{d}}$ School of Veterinary Medicine, Nihon University, Japan

e Neural Tissue Engineering, Keele School of Medicine, Keele University, Keele, UK

* Corresponding author

l.wong@bristol.ac.uk

Bristol Medical School, Dorothy Hodgkin Building, Whitson Street, Bristol BS1 3NY 


\section{Abstract}

Spinal cord injury (SCl) can cause chronic paralysis and incontinence and remains a major worldwide healthcare burden, with no regenerative treatment clinically available. Intraspinal transplantation of olfactory ensheathing cells (OECs) and injection of chondroitinase $A B C$ (chABC) are both promising therapies but limited and unpredictable responses are seen, particularly in canine clinical trials. Sustained delivery of chABC presents a challenge due to its thermal instability; we hypothesised that transplantation of canine olfactory mucosal OECs genetically modified ex vivo by lentiviral transduction to express chABC (COEC-chABC) would provide novel delivery of chABC and synergistic therapy. Rats were randomly divided into $\mathrm{COEC}-\mathrm{Ch} A B C, \mathrm{COEC}$, or vehicle transplanted groups and received transplant immediately after dorsal column crush corticospinal tract (CST) injury. Rehabilitation for forepaw reaching and blinded behavioural testing was conducted for 8 weeks. We show that $\mathrm{COEC}$-chABC transplanted animals recover greater forepaw reaching accuracy on Whishaw testing and more normal gait than COEC transplanted or vehicle control rats. Increased CST axon sprouting cranial to the injury and serotonergic fibres caudal to the injury suggest a mechanism for recovery. We therefore demonstrate that $\mathrm{COECS}$ can deliver sufficient chABC to drive modest functional improvement, and that this genetically engineered cellular and molecular approach is a feasible combination therapy for SCI. 


\subsection{Keywords}

Olfactory ensheathing cells;

Chondroitinase $A B C$;

Spinal Cord Injury;

Genetic engineering;

Combination therapy

\subsection{Abbreviations}

$\begin{array}{ll}\text { SHT } & \text { Serotonin } \\ \text { BBB } & \text { Basso, Beattie and Bresnahan } \\ \text { BDA } & \text { Biotinylated Dextran Amine } \\ \text { ChABC } & \text { Chondroitinase ABC } \\ \text { COEC } & \text { Canine mucosal Olfactory Ensheathing Cell } \\ \text { COEC-chABC } & \text { Canine mucosal Olfactory Ensheathing Cell expressing Chondrtoinase ABC and GFP } \\ \text { cOEC-GFP } & \text { Canine mucosal Olfactory Ensheathing Cell expressing GFP } \\ \text { CSPG } & \text { Chondroitin Sulphate Proteoglycan } \\ \text { CST } & \text { Corticospinal Tract } \\ \text { GFP } & \text { Green Fluorescent Protein } \\ \text { HBSS } & \text { Hank's Balanced Salt Solution } \\ \text { LV-chABC } & \text { Lentivirus expressing Chondroitinase ABC and GFP } \\ \text { LV-GFP } & \text { Lentivirus expressing GFP } \\ \text { OEC } & \text { Olfactory Ensheathing Cell } \\ \text { OB } & \text { Olfactory Bulb } \\ \text { OM } & \text { Olfactory Mucosa } \\ \text { SCI } & \text { Spinal Cord Injury } \\ \text { VGlut1 } & \text { Vesicular Glutamate Transporter 1 }\end{array}$




\section{Introduction}

Injury to the spinal cord can lead to permanent disability with life-changing consequences for those affected, with no regenerative treatment currently available. The pathophysiology of spinal cord injury (SCI) is complex and multifaceted (for reviews, see Dumont et al., 2001; Greenhalgh et al., 2020; Silva et al., 2014) and a combination of therapies is therefore likely to be required to facilitate axonal regeneration and enhance neuroplasticity (Griffin and Bradke, 2020).

Olfactory ensheathing cells (OECs) and chondroitinase $A B C$ (chABC) are two promising therapies for SCI. OECs have been shown to increase axonal regeneration (Li et al., 1998; Ramón-Cueto et al., 1998; Takeoka et al., 2011) and remyelination (Imaizumi et al., 1998; Smith et al., 2002), and improve locomotor outcomes in experimental rodent $\mathrm{SCl}$ as confirmed in two recent meta-analyses (Nakhjavan-Shahraki et al., 2018; Watzlawick et al., 2016). Autologous OECs can be safely obtained from the nasal olfactory mucosa (OM) of humans (Andrews et al., 2016) and companion dogs (Ito et al., 2019). This has facilitated phase 1 trials in humans demonstrating safety of OEC transplant (Li et al., 2015), and a randomised and blinded controlled trial in pet dogs with chronic thoracolumbar SCI which demonstrated improvements in fore-hindlimb coordination (Granger et al., 2012).

Chondroitin sulphate proteoglycans (CSPGs) contribute to glial scar formation after $\mathrm{SCl}$ and have a preponderant inhibitory effect on axon regeneration (Silver and Miller, 2004). Digestion of CSPGs by chABC allows axonal regeneration and increases neuroplasticity (Alilain et al., 2011; Bradbury et al., 2002; Moon et al., 2001), improving locomotion and recovery of skilled voluntary movement in multiple rodent models (Alilain et al., 2011; Bradbury et al., 2002; Caggiano et al., 2005; García-Alías et al., 2009). This positive effect is also seen in chronic injuries (Wang et al., 2011; Warren et al., 2018) and across species, reported in cats (Jefferson et al., 2011; Tester and Howland, 2008) and non-human primates (Bowes et al., 2012; Rosenzweig et al., 2019). In companion dogs, fore-hindlimb coordination was improved in a randomised controlled trial testing chABC (Hu et al., 2018).

Chondroitinase $A B C$ provides one of the best options for combination therapy due to its pleiotropic effects which can augment other therapies (Muir et al., 2019; Zhao and Fawcett, 2013). In the presence of $\mathrm{ch} A B C$, regenerating axons are able to create functional synapses with remaining spinal cord neurons (Houle et al., 2006; Steinmetz et al., 2005), a critical step required for recovery of voluntary function. OEC transplantation induces less astrocytic and CSPG response than other cell transplants, notably Schwann cells (Lakatos et al., 2003); combining reduction of inhibitory cues using chABC with creation of an environment favourable to axon regeneration using OECs may therefore provide synergistic effects. Supporting this concept, intraspinal OEC transplant combined with bolus 
chABC delivery by mini-pump showed a greater effect than cell transplant alone in preventing deterioration of bladder function (Fouad et al., 2009) and improving locomotor score after spinal cord transection in rats (Fouad et al., 2005).

A major barrier to the translation of intralesional chABC injection from lab to clinic is its rapid degradation at body temperature (Tester et al., 2007). In severe clinical injuries, delivery of chABC would need to be more sustained than is available after a single injection (Bradbury and Carter, 2011). Currently this would necessitate repeated spinal injections which are clinically undesirable, or intrathecal pump delivery. The latter, while successful in rodents (García-Alías et al., 2009; Wang et al., 2011), does not provide intra-parenchymal delivery that is important for larger animals (Rosenzweig et al., 2019). There is therefore a need for new delivery methods of chABC appropriate for clinical use. Previous approaches have included chemical thermostabilisation of chABC (Lee et al., 2010; Raspa et al., 2019), or gene therapy (Bartus et al., 2014; Burnside et al., 2018; James et al., 2015; Muir et al., 2010). Here we describe an alternative approach to delivering chABC - ex vivo genetic modification of OECs by lentiviral vector. This approach has been used in Schwann cells engineered to express chABC (Warren et al., 2020) or chABC and neurotrophin-3 (Kanno et al., 2014), and adds an additional level of safety compared to direct viral delivery of gene therapy making it more feasible for clinical use. We have previously reported that these modified canine OECS (COECs) express chABC in vitro (Carwardine et al., 2016) and can digest CSPGs (Carwardine et al., 2017).

In this study we perform a randomised and blinded controlled experiment with allocation concealment to test the efficacy of canine OECs expressing chABC ( $C O E C$-chABC) in improving recovery of forepaw reaching after acute dorsal column crush injury in rats, and compare this to COECs alone. This is the first functional test of $C O E C$-chABC. Rats were transplanted with either $C O E C$-chABC, $C O E C s$ expressing GFP only (cOEC-GFP) or vehicle control and all groups received home cage forepaw rehabilitation; an outline of the experimental timeline is shown in Supplementary Fig. 1. We show that transplantation of $C O E C-$ ch $A B C$ mediates sustained $c h A B C$ secretion and promotes sprouting of injured corticospinal tract (CST) axons and motor fibres to effect a greater degree of voluntary motor recovery than seen after either COEC transplant or vehicle control. 


\section{Materials and Methods}

\subsection{Cell culture}

Canine mucosal cOECs were obtained from a cell bank formed as part of a previous clinical trial (Granger et al., 2012), and retrieved from $-80^{\circ} \mathrm{C}$ storage. Briefly, cells were originally obtained by biopsy of the olfactory mucosa via a frontal sinus surgical approach, dissected free of blood vessels and non-olfactory mucosa, chopped and dissociated, then plated in fresh media as below. During subsequent passages, cells were differentially trypsinised into reduced-serum media to preferentially select COECs from contaminating fibroblasts (Granger et al., 2012; Ito et al., 2008). Cells were cultured in this experiment in a similar manner and as per previously published methods (Carwardine et al., 2016; Granger et al., 2012; Ito et al., 2008) on poly-I-lysine (PLL, Sigma) coated flasks in growth medium consisting of low glucose Dulbecco's Modified Eagle's Media (Sigma), 10\% foetal bovine serum (Gibco), 1\% gentamycin or penicillin and streptomycin (Sigma) and growth factors neuregulin-1

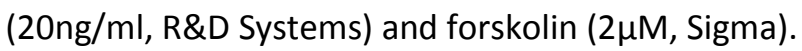

Cells were characterised prior to transplantation by immunocytochemistry as previously described (Carwardine et al., 2017) using antibodies for nerve growth factor receptor (p75) (MAB5364, Millipore; 1:200) to identify cOECs and fibronectin (AO245, DAKO; 1:400) to identify fibroblasts, the main culture contaminant.

\subsection{Lentiviral transduction}

The Proteus vulgaris chABC gene was previously modified to produce active chABC in mammalian cells (Muir et al., 2010). This chABC gene was codon optimised and subcloned into a third-generation selfinactivating non-replicating lentiviral plasmid: pRRL-SFFV-GFP-CMV-chABC (LV-chABC) (Carwardine et al., 2016), hence expressing chABC under a CMV (cytomegalovirus) promotor and GFP (green fluorescent protein) under the SFFV (spleen focus forming virus) promotor on a pRRL backbone. A lentiviral plasmid expressing just GFP (pRRL-CMV-GFP; LV-GFP) was used as control. At 10 days prior to transplant, cOECs were transduced with LV-GFP or LV-chABC at multiplicity of infection 10. Transduced cells were detected by immunohistochemistry using chicken anti-GFP (AB16667, Abcam; 1:2000). Secreted chABC was detected by Morgan Elson assay as previously described (Carwardine et al., 2016; Muir et al., 2010).

\subsection{Experimental groups}

Animals were transplanted with either COEC-ChABCs to test combination therapy, COEC-GFPs to control for transduction and to test COECs alone, or media solution to control for injection. Sham 
animals receiving cervical laminectomy surgery but not $\mathrm{SCl}$ were included to confirm the specific effect of dorsal column crush lesion in our lab and control for the effect of surgery.

We pre-specified our primary outcome measure for this study as number of pellets retrieved on Montoya staircase. Power calculations based on previously reported data for staircase retrieval after dorsal column crush injury and acute administration of chABC with forepaw rehabilitation (GarcíaAlías et al., 2009) predicted $n=6$ per group to show a statistically significant difference between groups at power $80 \%$, alpha $=0.05$.

A total of 53 rats were used, 45 for behavioural outcomes and 8 for analysis of cell survival and CSPG digestion at 2 weeks ( $n=4$ for COEC-GFP and COEC-chABC groups). Seven animals ( 3 cOEC-chABC, 1 COEC-GFP and 3 media transplanted) were culled three days after injury because they had persistent limb deficits impeding movement. One COEC-ChABC animal died under anaesthesia during BDA tracer injection surgery after week 6 behaviour data collection. Final group sizes were $n=9$ for cOEC-chABC group, $n=11$ for cOEC-GFP group, $n=9$ for media group, and $n=9$ for sham animals.

\subsection{Surgical procedures}

All procedures were licensed under the Animal Scientific Procedures Act (1996), reviewed by the University of Bristol Ethical Review Board, and performed in line with Home Office guidelines and regulations. Animals were housed in a temperature controlled specific-pathogen free facility with a 12:12 hour light:dark cycle in enriched cages on wood shavings with additional paper nesting material in groups of up to 4. Rats had ad lib access to water at all times and to food apart from where specified for behavioural testing.

Rats were randomly allocated to equal sized groups and the order of surgery randomised using random number generation. Surgeons were blinded to transplant group. Surgeries were performed in 2 batches with equal groups following the same experimental protocol. All animals were immunosuppressed with $15 \mathrm{mg} / \mathrm{kg} /$ day cyclosporine (Sandimmune, Novartis) from 24 hours prior to cell transplantation initially by daily intraperitoneal injection for 7 days, then by oral administration (Neoral, Novartis) in drinking water until termination.

\subsubsection{Dorsal column crush injury and cell transplant}

Adult male Wistar rats (Charles River) weighing between 225-250g were anaesthetised with intraperitoneal ketamine $(60 \mathrm{mg} / \mathrm{kg})$ and medetomidine $(250 \mu \mathrm{g} / \mathrm{kg})$. Lidocaine solution $(2 \%)$ was used on the surface of the spinal cord prior to injury and pain relief was provided by $0.02 \mathrm{mg} / \mathrm{kg}$ buprenorphine. A dorsal column crush lesion of the CST was performed as previously described 
(Bradbury et al., 1999; Carwardine et al., 2017) by performing a dorsal laminectomy at cervical vertebrae 3 then inserting marked forceps to a depth of $2 \mathrm{~mm}$ within the dorsal columns and crushing for 10 seconds. Sham animals received laminectomy but no injury to the spinal cord. Body temperature was monitored and maintained by use of a heat mat throughout surgery. Sedation was reversed with $50 \mu \mathrm{g} / \mathrm{kg}$ atipamezole after routine tissue and skin closure.

Cells were trypsinised, washed in Hank's Balanced Salt Solution (HBSS), resuspended at a concentration of 90,000 cells per $\mu \mathrm{L}$ and kept on ice until transplant. Cells were transplanted immediately after injury and within 90 minutes of trypsinisation. Cells were injected in the midline at a rate of $250 \mathrm{~nL} /$ minute using a microinjector (UMP Pump II, World Precision Instruments) in 2 injections each of volume $1 \mu \mathrm{L}$, cranial and caudal to the lesion. A total number of 180,000 cells were transplanted per rat. Media transplant animals received the same injections and volume of HBSS.

\subsubsection{BDA axon tracer injection}

After week 6 behavioural testing, all transplanted animals received a second surgery to inject $10 \%$ biotinylated dextran amine (BDA) (Sigma) into the sensorimotor cortex. Animals were anaesthetised and received pain relief as per previous surgery. They were placed in a stereotactic frame and a midline incision was made over the skull. Six stereotactic injections were made bilaterally with reference to bregma as previously reported (Carwardine et al., 2017), after drilling through the skull, at: (rostro-caudal/lateral in $\mathrm{mm}$ ) $-1.5 / 2.5,-0.5 / 3.5,+0.5 / 3.5,+1 / 1.5,+1.5 / 2.5,+2 / 3.5$. All $1 \mu$ injections were made $2 \mathrm{~mm}$ deep to the brain surface. Skin was closed and animals recovered routinely.

\subsection{Behavioural assessment}

All behavioural testing was performed by the same person blinded to treatment group. We prespecified pellet retrieval on Montoya staircase (Montoya et al., 1991), where rats reach down to collect reward pellets from different levels, as our primary outcome measure. Staircase testing was performed as previously described (García-Alías et al., 2008) and all animals were trained on this apparatus pre-surgery for 3 weeks, 5 days a week, once a day for 15 minutes. Number of pellets retrieved and accuracy of pellet retrieval (i.e. number of pellets retrieved as a percentage of total pellets displaced) was determined. Animals retrieving a minimum of 10 pellets per trial were included in behavioural analysis. Animals also received training in ladder crossing, crossing a 1 metre, regularly spaced ladder for reward pellets 3 times daily for 5 days prior to SCl surgery. Weekly behavioural testing of staircase and ladder ability after overnight starvation was performed twice weekly in the morning up to 8 weeks after injury. For each week, an average value of these two trials was used in analysis. Post-hoc, a comparison was made between the number of animals in each group who 
increased pellet retrieval by a 'clinically significant' amount (determined empirically as an increase of 5 pellets, approximately a $100 \%$ increase in ability from immediately post-injury).

Whishaw reaching was performed using an apparatus as previously described (Whishaw et al., 1993) requiring animals to reach through a $1.5 \mathrm{~cm}$ slit onto a raised platform to retrieve reward pellets. This was pre-specified to be conducted once, at 6 weeks after injury, without prior training as previously described (García-Alías et al., 2009). Animals were initially placed in the box for 10 minutes with reward pellets placed at the opening to habituate them, then the number of pellets retrieved and displaced on first reaching attempt over a subsequent 5 minute period was recorded.

Gait analysis was performed by dipping each animal's forepaws in ink, then allowing them to walk along a $1 \mathrm{~m}$ paper covered runway. Paw width was measured between the outside edges of the $2^{\text {nd }}$ and $5^{\text {th }}$ digits of the first distinguishable print. Stride length and base of support was measured from the middle back of each print and averaged over the first 5 consecutively distinguishable prints. Base of support is the perpendicular lateral distance between consecutive prints and a measure of lateral stability.

All animals received rehabilitation specific for forepaw reaching as previously described (García-Alías et al., 2009). Briefly, grids with apertures $1.7 \times 1.7 \mathrm{~cm}$ wide and $2.2 \mathrm{~cm}$ deep were filled with seeds and other rewards and placed in home cages 5 days a week for 1 hour a day from 1 week after injury. Animals could only reach rewards with their forepaws, and cages were intermittently monitored during the hour to confirm all animals were reaching for pellets.

A summary of the experimental timeline is shown in Supplementary Fig. 1.

\subsection{Histology}

Rats were euthanised by terminal anaesthesia with pentobarbitone $(1 \mathrm{ml} / \mathrm{kg}$ intraperitoneal) and transcardially perfused with PBS followed by 4\% PFA. The relevant (cervical) spinal cord was dissected and mounted in $10 \% \mathrm{w} / \mathrm{v}$ gelatin (Sigma) in PBS then fixed in PFA overnight. Cords and gelatin were transferred to $30 \%$ sucrose in PBS for 48 hours or until equilibrated, then transferred to optimised cutting complex (TissueTek) and snap frozen at $-80^{\circ} \mathrm{C}$. Samples were sectioned free floating on a cryotome at $30-40 \mu \mathrm{m}$; the central area around the lesion $( \pm 10 \mathrm{~mm})$ was sectioned dorsally while $2 \mathrm{~mm}$ segments further cranial and caudal to this were sectioned transversely.

Immunohistochemistry was performed as previously described (Carwardine et al., 2017) using the following primary antibodies: rabbit anti-5HT (Serotonin; Immunostar 20080, 1:10000) to label 
serotonergic neuronal fibers; rabbit anti-vGlut1 (Synaptic Systems 135303, 1:500) to label presynaptic glutamatergic proprioceptive afferents; PKCX (Santa Cruz SC-211, 1:1000) to visualise the CST and verify injuries were consistent and completely disrupted the CST in dorsal column crush lesions. BDA labelled sections were incubated overnight at $4^{\circ} \mathrm{C}$ with Avidin-Neutravadin Texas Red (Thermofisher, 1:500) for fluorescent imaging.

To determine chABC activity, immunohistochemistry using 3-3' diaminobenzidine (DAB) staining was performed with Vectastain Elite $A B C$ kit (VectorLabs) according to manufacturer's protocol using mouse anti-C4S (MP Biomedicals 6365111, 1:5000) to label digested stubs of CSPG degradation by chABC and mouse anti-neurocan (DSHB IFC-c, 1:50) to label a subset of CSPGs. Slides were serially dehydrated in 70, 90 and 95\% ethanol for 1 minute each followed by 2 minutes in xylene before coverslips were mounted using DPX mounting media (Sigma 06522).

\subsection{Image Analysis}

\subsubsection{Quantification of cell survival in vivo}

Serial dorsal sections (1 in 10) were processed for p75 and GFP as per immunohistochemistry and transplanted cells were identified by GFP. Given the relatively small number of surviving cells, all transplanted cells in a section were counted manually by an observer blind to transplant group. Total cell count was multiplied by 10 to give an estimate of total surviving transplanted cells in the cord, and this was expressed as a percentage of transduced (GFP+) cells transplanted as previously reported (Carwardine et al., 2017).

\subsubsection{CST axon tracing imaging and analysis}

Three serial (1 in 10) dorsal sections though the lesion incorporating the CST were imaged at 20x using a motorised stage and tilescan software (Leica DMI6000 inverted epifluorescence microscope with Leica DFC365FX monochrome digital camera and Leica LAS-X acquisition software). Images were processed using the FeatureJ plugin (Meijering, 2003) of Fiji ImageJ (Schindelin et al., 2012) to selectively segment and threshold linear structures using Hessian-based edge detection (Grider et al., 2006). Three defined regions of interest were traced manually within ImageJ for each section: (i) white matter; (ii) grey matter cranial to the lesion edge; and (iii) white matter caudal to the lesion edge. All regions of interest were of length $2 \mathrm{~mm}$ from the lesion. The percentage of BDA positive pixels within each region of interest was quantified for each region (referred to as BDA pixel density). Cranial grey matter and caudal white matter pixel densities were expressed as a ratio to the cranial white matter pixel density (to normalise for variation in BDA staining between animals and sections (Grider et al., 2006)). 


\subsubsection{Quantification of immunostaining}

Five transverse cranial and caudal sections for each animal were immunostained as above for vGlut1 and serotonin $(5 \mathrm{HT})$. Grey matter laminae and white matter regions were defined by custom made templates produced in InkScape (The Inkscape Project, v0.92). Merge whole-section tilescan images were imported into Fiji ImageJ and transformed by unwarping with reference to points annotated on each image and template at clear landmarks (e.g. central canal, dorsal and ventral points of grey matter, 12-18 points per image) using a custom macro written for the Bristol Wolfson Imaging Modular Image Analysis Plugin (Cross, n.d.). The number of positive pixels above a set manual threshold was expressed as a percentage (pixel density) of total pixels within each region.

\subsection{Statistical Analysis}

All data was tested for normal distribution using the Shapiro-Wilk test. Parametric data was analysed using t-tests or one-way ANOVA with post-hoc Tukey test unless otherwise specified. Non-parametric data was analysed by Mann-Whitney $U$ or Kruskall-Wallis. Behavioural testing with repeated measures (staircase and ladder testing) was analysed using linear mixed effects model analysis in R (R Core Team, 2013) with fixed effects defined as transplant group and time, and individual animal assigned as a random effect. Post-hoc Tukey multiple comparison test was used to compare groups. All other statistical analysis was performed using Graphpad Prism v7. 


\section{Results}

\subsection{Characterisation of transplanted canine OEC population}

The canine mucosal COEC population was characterised before transplantation by immunofluorescence staining with virtually no contaminant fibroblasts identified (cOEC proportion $99.3 \pm 0.4 \%$, Fig. $1 \mathrm{~A})$. Quantification of GFP+ transduced cells showed similar transduction rates in COEC-chABC and COEC-GFP groups, with $52.2 \pm 13.9 \%$ and $45.8 \pm 8.7 \%$ GFP + respectively (Fig. $1 \mathrm{~B} ; \mathrm{p}=0.33$ unpaired t-test). We confirmed secretion of active chABC from transduced COEC-chABCs prior to transplant and negligible chABC from cOEC-GFPs by Morgan Elson reaction (Fig. 1C).

\subsection{COEC-chABC survive and secrete active chABC after transplant}

At 2 weeks after transplant of the transduced canine COECs into the rat spinal cord lesion, both COECGFP and COEC-chABC could be identified on spinal cord sections by immunofluorescent staining for GFP (Fig. 2A). Quantification of surviving cells, expressed as a percentage of transplanted GFP+ cells, showed no significant difference ( $p=0.25$, unpaired t-test) between COEC-GFP and COEC-chABC survival (Fig. 2 B) with $0.95 \pm 0.73 \%$ cOEC-chABC ( 1710 cells) and $0.45 \pm 0.27 \%$ cOEC-GFP ( $\sim 810$ cells) present $(n=4)$.

Transplanted COEC-chABC secrete active $C h A B C$, as evidenced by the decreased neurocan immunostaining and presence of digested stubs of CSPGs localised around the cystic lesion and area of COEC transplant in COEC-chABC animals (Fig. 2C). No comparable digestion was observed in COECGFP transplanted animals. Digested stubs of CSPG digestion (C4S) were further seen at 9 weeks, but this was less extensive that at the 2 week time-point and we did not see clear absence of neurocan staining.

Complete injuries to the CST were confirmed in all animals by PKCX staining (Supplementary Fig. 2). We saw few COECs at 9 weeks after transplant and no evidence of tumour formation.

\subsection{Transplanted COEC-chABC promote forepaw reaching recovery}

\subsubsection{Montoya Staircase}

All animals that received $\mathrm{SCl}$ showed a consistent decrease in number of pellets retrieved on Montoya Staircase task at week 1 after injury compared to baseline (pre-injury) levels (Fig. 3A). The number of pellets retrieved over the follow-up period was not significantly different between transplant groups (Fig. 3A). There was a significant effect of experimental group $[F(3,34)=9.7, p<0.0001$; mixed effects analysis] as all transplant groups were significantly different to sham animals ( $p=0.033$ for cOECchABC, $p=0.0005$ for cOEC-GFP and $p=0.0002$ for media animals; post-hoc Tukey test). There was also 
a significant effect of time $[F(7,237)=10.5, p<0.0001]$ and an interaction effect between time and group $[F(21,237)=7.7, p<0.0001]$.

COEC-chABC transplanted animals were the only transplant group to significantly increase the number of pellets retrieved (from $6.1 \pm 3.5$ at week 1 to maximum $10.4 \pm 3.6$ at week $6 ; p=0.036$ post-hoc Tukey test). COEC-GFP and media transplanted animals did not significantly increase number of pellets retrieved at any time point (from $5.9 \pm 4.8$ to maximum 7.5 $\pm 5.7, p=0.90$ and $5.4 \pm 3.5$ to maximum $6.7 \pm 4.2, p=0.98$ respectively at week 6 ). Additionally, a higher proportion of COEC-chABC animals showed improvements in the Montoya staircase test, with more animals able to retrieve a minimum of five more pellets at week 6 than week 1 ( 4 of $9,44 \%$ ), compared to cOEC-GFP transplanted animals ( 2 of $11,18 \%$ ) and media controls (0 of 9, 0\%) (Fig. 3B).

The accuracy of pellet retrieval on Montoya Staircase over the follow-up period was not significantly different between transplant groups (Fig. 3C). There was a significant effect of experimental group $[F(3,34)=5.4, p<0.0038$; mixed effects analysis] as all transplant groups were significantly different to sham animals ( $p<0.0001$ for all groups, post-hoc Tukey test). There was a significant effect of time $[F(5,159)=10.6, p<0.0001]$ and an interaction effect between time and group $[F(21,236)=3.1$, $p<0.0001]$.

\subsubsection{Whishaw Reaching}

We further tested forepaw reaching using the Whishaw reaching task (Fig. 3D). cOEC-chABC transplanted animals had a significantly greater accuracy of retrieval $(61.7 \pm 9.2 \%)$ compared to cOECGFP (39.1 \pm 7.3\%; $F(3,19)=14.8, p<0.0001$ one-way ANOVA, $p=0.01$ post-hoc Tukey test) and media $(34.2 \pm 9.9 \%, p=0.0013)$ transplanted animals (Fig. $3 C$ ). cOEC-chABC transplanted animals performed similarly to sham animals $(62.9 \pm 10.2 \%)$, who also showed greater accuracy than media $(p=0.0001)$ and cOEC-GFP $(p=0.0015)$ transplanted animals.

\subsection{Transplanted COEC-chABC improves gait but not ladder crossing}

Animals had few foot slips when crossing a horizonal ladder prior to $\mathrm{SCl}$ and all animals showed an increased number of foot slips after injury which decreased over the 8 weeks of testing (Fig. 4A). A significant effect of group $[F(3,32)=28.1, p<0.0001)]$ and time $[F(7,185)=41.0, p<0.0001]$ was seen but there was no significant difference between transplant groups ( $p>0.05$ on post-hoc Tukey test). All transplant groups were significantly different to sham animals ( $p<0.0001$ for all groups).

Gait analysis (illustrated in Fig. 4B) showed COEC-chABC transplanted and sham animals had similar stride lengths (Fig. 4C, $(277 \pm 29 \mathrm{~mm}$ and $280 \pm 44 \mathrm{~mm}$ respectively) which were significantly longer than 
media transplanted animals $(213 \pm 63 \mathrm{~mm},[F(3,33)=4.9, p=0.0062$ one-way ANOVA; $p=0.03$ and $p=0.023$ respectively on post-hoc Tukey test). Base of support was narrower in COEC-chABC animals $(13.4 \pm 5.9 \mathrm{~mm})$ than COEC-GFP $(22.1 \pm 7.1 \mathrm{~mm})$ and media $(21.6 \pm 5.8 \mathrm{~mm})$ transplanted animals (Fig. 4D) $[F(3,33)=4.1, p=0.015$ one-way ANOVAs; $p=0.019$ and $p=0.041$ respectively on post-hoc Tukey test $]$, and not significantly different to sham animals $(17.3 \pm 4.6 \mathrm{~mm})$. No significant difference in paw width was seen between groups (Fig. 4E).

\subsection{Transplanted COEC-chABC increases CST and 5HT sprouting}

\subsubsection{CST axon response to transplant}

CST axon sprouting was determined across the grey-white matter border cranial to the lesion by BDA labelling (Fig. 5A). Significantly more BDA positive axons were observed sprouting into the grey matter in $\operatorname{COEC}$-chABC transplanted animals compared to both media and cOEC-GFP $[F(2,25)=12.9, p=0.0001$ one-way ANOVA, $p=0.0004$ and $p=0.0005$ post-hoc Tukey respectively] (Fig. 5B). Occasional BDA positive CST axons were seen caudal to the lesion but no significant difference was seen between groups caudal to the injury site (Fig. 5C).

\subsubsection{Serotonergic and vGlut1 axonal response to transplant}

Examination of serotonergic fibres in transverse sections $10 \mathrm{~mm}$ caudal to the lesion (Fig. 6A) revealed that there was an increased number of fibres across multiple regions of grey and white matter in COECchABC transplanted animals compared to COEC-GFP and media transplanted animals. 5HT immunostaining was analysed in different regions (Fig. 6B) and found to be significantly greater for the COEC-chABC group in dorsal (Fig. 6D; $p=0.026$ Kruskall-Wallis, $p=0.036$ Dunn's post-hoc test) and ventral grey matter (Fig. $6 \mathrm{E} ; \mathrm{p}=0.0068$ Kruskall-Wallis, $\mathrm{p}=0.020$ Dunn's post-hoc) as well as ventral funiculi (Fig. 6F; $F(3,12)=8.0, p=0.0040$ one-way ANOVA, post-hoc Tukey test $p=0.0067$ compared to media, $p=0.0076$ compared to COEC-GFP). A similar but non-significant change was seen in lateral funiculi ( $p=0.021$ Kruskall-Wallis, $p=0.0502$ Dunn's post-hoc) (Fig. $6 C$ ).

Similar analysis of vGlut1 immunostaining $10 \mathrm{~mm}$ cranial and caudal to the lesion showed no significant differences between transplant groups in any region (Supplementary Fig. 3). 


\section{Discussion}

This study provides the first evidence that $\mathrm{COEC}-\mathrm{ch} A B C$ can mediate greater functional improvement than $\mathrm{COECs}$ alone and deliver sufficient $\mathrm{ChABC}$ to improve recovery of voluntary limb function in the presence of COECs and alongside forepaw reaching rehabilitation. COEC-chABC may therefore represent a practicable combination therapy for $\mathrm{SCl}$ and novel delivery method of chABC.

The most common human $\mathrm{SCl}$ today is an incomplete cervical injury without associated fracture or dislocation (Badhiwala et al., 2019), with recovery of arm and hand function described by tetraplegics as most desirable (Anderson, 2004). Cervical dorsal column crush SCl in rats produces forepaw reaching deficits by selectively destroying the dorsal CST, the main tract responsible for control of voluntary movements in humans (Welniarz et al., 2017). If motor function is to be improved after human SCl then regeneration of this tract is crucial (Blesch and Tuszynski, 2009). High levels of homology between rodent forepaw and human hand movements during reaching and grasping suggest the dorsal column crush model is a good model of hand function (Sacrey et al., 2009).

Prior studies have shown chABC to be effective at improving forepaw recovery using a range of delivery methods including bolus delivery of chABC through intrathecal catheter over 10 days (Bradbury et al., 2002; García-Alías et al., 2009, 2008; Wang et al., 2011), intraparenchymal lentiviral injection leading to long-term expression of chABC (James et al., 2015), and most recently with a doxycycline regulatable viral vector (Burnside et al., 2018). Using COEC-chABC, the time-period over which chABC is delivered is dependent on transplanted COEC survival. We have demonstrated here that transplanted modified canine OECs (COEC-GFP and COEC-chABC) survive a minimum of 2 weeks after xenotransplant into cervical dorsal column crush injuries. Positive C4S staining and loss of neurocan staining at 2 weeks confirms CSPG digestion and active chABC during these 2 weeks. This is therefore comparable to previously reported efficacious chABC delivery strategies.

We further see evidence of CSPG digestion at 9 weeks (positive C4S staining), however neurocan staining is not reduced at this time point. C4S stubs remain present in the spinal cord for up to 4 weeks (Brückner et al., 1998), while neurocan begins to return 7 days after chABC administration (Lin et al., 2008), which suggests active chABC may have been secreted by COECs up to at least 4 weeks in our study. This would fit the expected time course of COEC death from other studies, which report the majority of cell loss within the first week after transplant followed by a more gradual tailing off of cell numbers (Khankan et al., 2016; Li et al., 2010; Pearse et al., 2007). Future work to include histological analysis at a series of time-points would be valuable to further characterise $\mathrm{COEC}$ survival and ChABC expression at intermediate time-points. 
We see improvements in forepaw reaching and gait appearing at 6-8 weeks. This suggests that recovery is due to regeneration and/or plasticity rather than an effect of neuroprotection as is also reported after administration of chABC (Bartus et al., 2014; Bradbury and Carter, 2011), although neuroprotective effects were not explored in detail in this study. Recovery at time-points beyond the likely period of chABC delivery is consistent with prior reports where chABC is believed to create a window of opportunity for plasticity and reorganisation, and forepaw function continues to increase beyond this time (Burnside et al., 2018; García-Alías et al., 2009, 2008). Indeed, our forepaw reaching results are comparable to a previous study by García-Alías et al. (2009) where forepaw reaching in rats was tested following the same dorsal column crush injury paradigm with specific rehabilitation \pm chABC administration via 10-day intrathecal pump delivery. The number of pellets retrieved on Montoya Staircase $(9.5 \pm 1.0$ reported for pump delivered ChABC and $10.4 \pm 3.6$ for our COEC-chABC transplants, at week 6$)$ and accuracy of pellet retrieval on Whishaw reaching $(37.4 \pm 6 \%$ reported for pump delivered chABC and $61.7 \pm 9 \%$ for our COEC-chABC transplants) were similar or higher for COECchABC compared to values reported by García-Alías et al.

It is interesting to note that we do not see a difference in ladder crossing between transplant groups, which all received rehabilitation specific to forepaw reaching. The ladder crossing task is a less specific test of the CST, most likely involving spinocerebellar pathways, but a reduction in foot slips on ladder or beam walking has been reported previously for animals treated with chABC who received no rehabilitation (Bradbury et al., 2002; Burnside et al., 2018; James et al., 2015). General rehabilitation, but neither specific rehabilitation for forepaw reaching nor chABC administration alone, reduced ladder slips in another study (García-Alías et al., 2009). It has also been reported that rats receiving specific rehabilitation for forepaw reaching after $\mathrm{SCl}$ with no other intervention increased foot slips on ladder testing compared to animals receiving no rehabilitation (Girgis et al., 2007). This highlights the possibility that specific rehabilitation may have no, or possibly a negative, effect on 'untrained' behaviours, perhaps due to rehabilitation driving functional reorganisation within the spinal cord only towards trained behaviours. In our study, this process may have been enhanced by chABC induced sprouting and increased plasticity, to the detriment of untrained behaviours (for a review, see GarcíaAlías and Fawcett, 2012). Our study therefore re-iterates the importance of rehabilitation after SCI and ensuring training is designed for the desired outcome (Girgis et al., 2007; Tetzlaff et al., 2009), particularly in the presence of chABC.

Axon sprouting following chABC administration after $\mathrm{SCl}$ is a consistent finding across different models of injury and delivery methods (Bartus et al., 2014; Bradbury et al., 2002; Burnside et al., 2018; GarcíaAlías et al., 2009, 2008) and we also see clear increased sprouting of BDA stained axons cranial to the 
lesion with $\mathrm{COEC}-\mathrm{ch} A B C$ transplant after dorsal column crush injury. We see no significant difference in BDA positive CST axons caudal to the lesion. The CST is known to be particularly refractory to treatment (Blesch and Tuszynski, 2009; Tuszynski and Steward, 2012) and it is possible that the improved forepaw reaching and gait we recorded is a result of plasticity and compensatory reorganisation of neighbouring tracts not labelled with BDA. Indeed this compensation has previously been suggested in the rubrospinal (Jefferson et al., 2011; Kennedy, 1990) and reticulospinal (Filli et al., 2014) tracts. Increased levels of $5 \mathrm{HT}$ caudal to the lesion in the COEC-chABC transplanted animals after dorsal column crush injury further suggests non-specific re-organisation or regeneration of other motor tracts and has previously been reported after chABC delivery (Barritt et al., 2006; Bartus et al., 2014; García-Alías et al., 2009; Karimi-Abdolrezaee et al., 2010).

The lack of change in vGlut1 we report may also reflect this re-organisation. The refractory CST contains high levels of vGlut1 and low levels of vGlut2 (97\% and $1 \%)$ compared to the rubrospinal tract (97\% vGlut2 and $0.3 \%$ vGlut1) and reticulospinal tract (70\% vGlut2 and 4\% vGlut1) (Du Beau et al., 2012). If there were changes in the rubrospinal or reticulospinal tract these may therefore not be apparent on vGlut1 analysis. vGlut1 is also associated with primary myelinated sensory afferents from muscle mechanoreceptors (Alvarez et al., 2004; Todd et al., 2003) and proprioceptive afferents (Rotterman et al., 2014). The lack of change in vGlut1 may therefore also underlie the differential behavioural changes we see with greater improvement in Whishaw reaching (where vision may provide an additional trigger for movement and provide a compensatory mechanism for a lack of proprioception) compared to Montoya Staircase reaching (where conscious proprioception is required to trigger movement as the animal is reaching down to retrieve pellets that are out of sight).

We see no evidence for efficacy of COEC transplant alone (COEC-GFP group) in this xenotransplant of canine mucosal OECs into immunosuppressed rats. Two meta-analyses have demonstrated OECs improve recovery after $\mathrm{SCl}$ in rodent models with minimal bias in the literature (Nakhjavan-Shahraki et al., 2018; Watzlawick et al., 2016) giving clear evidence for efficacy of OECs. However, there are a number of exceptions to this with several studies failing to show behavioural improvements after OEC transplant (Bretzner et al., 2010, 2008; Deumens et al., 2006; Ruitenberg et al., 2005). This includes a direct replication study (Steward et al., 2006) of olfactory lamina propria transplant 1 month after rat spinal cord transection, which failed to show the behavioural improvement or serotonergic regeneration seen in the original study (Lu et al., 2002).

Axon regeneration of the CST and of $5 \mathrm{HT}$ fibres has often been reported after OEC transplant (Li et al., 1997; Nash et al., 2002; Ramón-Cueto et al., 2000; Roet and Verhaagen, 2014) but like behavioural recovery, there is a mixed picture in the literature with CST recovery especially not always responsive 
to OEC transplant (Deumens et al., 2006; Ruitenberg et al., 2005; Steward et al., 2006) even when functional recovery is seen (Guest et al., 2008). It is worth noting that the evidence specifically for forepaw reaching after cervical injury is particularly mixed, with marked heterogeneity in study design (e.g. blinding, allocation concealment, randomisation; for a review see Granger et al., 2014).

There are a large number of variables associated with OEC transplant experiments that might account for some these differences, namely: type of cells (olfactory mucosa or olfactory bulb), species of cells (allograft or xenograft), type of injury model (e.g. transection, crush, contusion), time of transplant (acute, sub-acute or chronic), transplantation parameters (e.g. volume and speed of injection), and even passage of transplanted cells. There are few studies which directly compare and assess these individual parameters, which make teasing out the impact of each one very difficult. It is generally accepted that regenerative outcomes are poorer in chronic transplants and with increasing passage of cells (Novikova et al., 2011; Pastrana et al., 2007), for reviews see (Nakhjavan-Shahraki et al., 2018; Reshamwala et al., 2019; Watzlawick et al., 2016). Further studies providing direct experimental comparison of individual parameters in the same model of injury and with standardised outcome measures would be hugely beneficial.

The quality of evidence for behavioural studies is also variable, particularly in older studies. In a metaanalysis of OEC transplant, only 28 out of 53 studies report blinding and only 17 report random allocation to groups (Watzlawick et al., 2016). It is well established that these factors can affect the outcomes seen through unconscious biases; across stem cell transplant studies for SCl, studies with blinded assessment of outcome had a significantly lower effect size (23.6\% compared to $30.3 \%)$ than those not blinded (Antonic et al., 2013). We report results of a study with allocation concealment, randomisation of group allocation and blinding of surgery, transplant and behavioural outcomes.

Mucosal cOECs were used in this study due to their clinical applicability, ease of sampling and safety profile (Andrews et al., 2016; Granger et al., 2012; Ito et al., 2019; Li et al., 2015) compared to olfactory bulb (OB) OECs where biopsy for cell harvesting has been associated with seizures (Andrews et al., 2016; Jeffery et al., 2005; Sarnat and Flores-Sarnat, 2016). There is debate around the comparative efficacy of $O B$ and $O M$ cells in the literature. Differences have been established in gene expression profile (Guérout et al., 2010) and phenotype (Roloff et al., 2013) but again very few studies directly compare the two populations in the same transplant experiment (Ibrahim et al., 2014; Mayeur et al., 2013). One study suggests minimal difference in electrophysiological and locomotor recovery between OB and purified OM OECs (Mayeur et al., 2013) while the other suggests greater recovery of climbing ability after dorsal root rhizotomy with OB OECs, but compares OM and OB data collected at different times (Ibrahim et al., 2014). Meta-analyses comparing between different studies corroborate 
these findings showing either only a slight trend to greater recovery with OB OECs (Watzlawick et al., 2016) or no difference in effect sizes (Nakhjavan-Shahraki et al., 2018). The risk:benefit ratio may therefore currently be in favour of the use of OM OECs in clinical transplants.

Low and variable OEC survival is commonly seen after experimental OEC transplant, with approximately 1000 OECs reported surviving at 3 weeks after transplant (Zhang et al., 2015) and a range of survival at between 1 and 4 weeks of $0.47-6.5 \%$ (for a review, see Reshamwala et al., 2019). We used xenograft (canine) cells in rats, reporting the first behavioural results after canine OEC transplant into rat $\mathrm{SCl}$. Although our survival rates are comparable to the literature there is some evidence that xenograft OECs survive less well than allograft OECs, and that xenotransplants may not survive as well in cyclosporine A immunosuppressed rats as athymic rats (Smith et al., 2002). It is interesting to note that COEC-ChABC cells in this study showed a trend to higher survival than COECGFP, although cell survival was variable and the difference is not statistically significant. It is possible that chABC could improve OEC adhesion and integration into sites of $\mathrm{SCl}$, as recently reported for Schwann cells (Warren et al., 2020). This could provide a mechanism for a synergistic effect of chABC and OECs and future work to characterise this further in vitro and in vivo would be justified.

There is continued difficulty in the clear identification of OECs and their discrimination from Schwann cells as they share many markers, including p75 (Bock et al., 2007; Ulrich et al., 2014). We, like others, have considered that the high proportion of OECs in olfactory mucosa and the removal during biopsy processing of blood vessels means that the vast majority of p75+ cells will be OECs. In the absence of a specific marker this approach makes the assumption that biopsy of the olfactory mucosa is the most likely source of OECs for clinical transplant and that this provides a "therapeutic transplant" (Ito et al., 2019; Kawaja et al., 2009). We cannot entirely discount the possibility that Schwann cells are present in our transplants but have used this cell population to prove concept for modification of a clinically relevant cell transplant population to express chABC.

Dose and quantity of chABC delivered will be dependent on COEC-chABC survival in this paradigm and it has been suggested that higher doses and/or longer administration of chABC provides greater efficacy (for a review, see Muir et al., 2019). We demonstrate there is sufficient chABC delivered in this model to mediate functional improvement but it is worth noting that autologous transplant of cells, as would be used in clinical trials (Feron et al., 2005; Granger et al., 2012; Lima et al., 2010, 2006; Mackay-Sim et al., 2008; Tabakow et al., 2013), would likely result in higher cell survival than we see in this study due to reduced graft rejection. Consequently, increased chABC delivery might be expected. 
Testing this novel COEC-chABC therapy in an autologous model - for example the canine clinical translational model (Granger et al., 2012; Jeffery et al., 2006; Moore et al., 2017) - could therefore be an important next step for COEC-chABC, made possible by the evidence presented in this study. Improving cell survival is also a key focus of a number of regenerative therapies using cell transplantation and delivery within protective hydrogel matrices is a promising approach (Ballios et al., 2015; Führmann et al., 2016) which could be readily added to this COEC-chABC combination therapy (Adams et al., 2019; Prager et al., 2020).

Ex vivo transduction of COECs adds an additional layer of safety (Naldini, 2011) compared to intraparenchymal viral delivery; obviating the need to expose host tissue to 'active' vector and providing an additional ability to screen transduced COECs in culture for replication competent lentivirus or abnormal proliferation of transduced cells before transplant in vivo. There are a number of clinical trials in progress using this approach of ex vivo cell transduction with third-generation replicationdeficient lentiviral constructs, particularly for leukaemias (Milone and O'Doherty, 2018), demonstrating the clinical feasibility of this approach. Temporally controllable delivery of chABC would further limit concerns and it is likely that chABC would only be required to form a window of neuroplasticity, particularly potent in association with rehabilitation to strengthen functional connections (García-Alías et al., 2009). A future combination therapy could be to use the recently reported doxycycline inducible system (Burnside et al., 2018) to transduce cOECs ex vivo before transplant within a protective hydrogel matrix.

In conclusion, we show moderate functional recovery of voluntary movement mediated by canine OECs expressing chABC after transplant in cervical dorsal column crush injury in rats receiving forepaw reaching rehabilitation. We therefore demonstrate proof-of-principle that $\mathrm{COECs}$ expressing chABC could provide a novel delivery method of $\mathrm{chABC}$ and combination therapy for $\mathrm{SCl}$. Future work will determine if this functional benefit extends to recovery of locomotion, after delayed transplant and in more severe injury models including the autologous canine clinical model. 


\section{Acknowledgments}

The authors gratefully acknowledge the Wolfson Bioimaging Facility, University of Bristol for their support and assistance in this work.

\section{Funding}

The authors would like to thank The Langford Trust for Animal Health and Welfare who generously funded this work through a grant awarded to NG, LFW and DC. They would also like to thank the Great Britain Sasakawa Foundation for Butterfield Award B87 awarded to NG and DI.

\section{Declaration of Competing Interests}

The authors declare no competing interests.

\section{Data Availability}

The data used in this study are available from the authors on request.

\section{Author Contributions}

Conceptualization, N.G., L.W.; Methodology, J.P, D.R.C., L.W., N.G.; Investigation, J.P., D.I., D.R.C., P.J., L.W.; Resources, D.M.C., N.G., L.W.; Writing - Original Draft, J.P.; Writing - Review \& Editing, J.P., D.I., D.R.C., D.M.C., N.G., L.W.; Supervision, D.M.C., N.G., L.W.; Funding Acquisition, D.I., D.M.C., N.G., L.W. 


\section{References}

Adams, C.F., Delaney, A.M., Carwardine, D.R., Tickle, J., Granger, N., Chari, D.M., 2019. NanoparticleBased Imaging of Clinical Transplant Populations Encapsulated in Protective Polymer Matrices. Macromol. Biosci. 19, 1800389. https://doi.org/10.1002/mabi.201800389

Alilain, W.J., Horn, K.P., Hu, H., Dick, T.E., Silver, J., 2011. Functional regeneration of respiratory pathways after spinal cord injury. Nature 475, 196-200. https://doi.org/10.1038/nature10199

Alvarez, F.J., Villalba, R.M., Zerda, R., Schneider, S.P., 2004. Vesicular Glutamate Transporters in the Spinal Cord, with Special Reference to Sensory Primary Afferent Synapses. J. Comp. Neurol. 472, 257-280. https://doi.org/10.1002/cne.20012

Anderson, K.D., 2004. Targeting Recovery: Priorities of the Spinal Cord-Injured Population. J. Neurotrauma 21, 1371-1383. https://doi.org/10.1089/neu.2004.21.1371

Andrews, P.J., Poirrier, A.-L., Lund, V.J., Choi, D., 2016. Safety of human olfactory mucosal biopsy for the purpose of olfactory ensheathing cell harvest and nerve repair: a prospective controlled study in patients undergoing endoscopic sinus surgery. Rhinology 54, 183-91. https://doi.org/10.4193/Rhin15.365

Antonic, A., Sena, E.S., Lees, J.S., Wills, T.E., Skeers, P., Batchelor, P.E., Macleod, M.R., Howells, D.W., 2013. Stem cell transplantation in traumatic spinal cord injury: a systematic review and metaanalysis of animal studies. PLoS Biol. 11, e1001738. https://doi.org/10.1371/journal.pbio.1001738

Badhiwala, J.H., Ahuja, C.S., Fehlings, M.G., 2019. Time is spine: A review of translational advances in spinal cord injury. J. Neurosurg. Spine. https://doi.org/10.3171/2018.9.SPINE18682

Ballios, B.G., Cooke, M.J., Donaldson, L., Coles, B.L.K., Morshead, C.M., van der Kooy, D., Shoichet, M.S., 2015. A Hyaluronan-Based Injectable Hydrogel Improves the Survival and Integration of Stem Cell Progeny following Transplantation. Stem cell reports 4, 1031-45. https://doi.org/10.1016/j.stemcr.2015.04.008

Barritt, A.W., Davies, M., Marchand, F., Hartley, R., Grist, J., Yip, P., McMahon, S.B., Bradbury, E.J., 2006. Chondroitinase $A B C$ promotes sprouting of intact and injured spinal systems after spinal cord injury. J. Neurosci. 26, 10856-67. https://doi.org/10.1523/JNEUROSCI.2980-06.2006

Bartus, K., James, N.D., Didangelos, A., Bosch, K.D., Verhaagen, J., Yáñez-Muñoz, R.J., Rogers, J.H., Schneider, B.L., Muir, E.M., Bradbury, E.J., 2014. Large-scale chondroitin sulfate proteoglycan digestion with chondroitinase gene therapy leads to reduced pathology and modulates macrophage phenotype following spinal cord contusion injury. J. Neurosci. 34, 4822-36. https://doi.org/10.1523/JNEUROSCI.4369-13.2014

Blesch, A., Tuszynski, M.H., 2009. Spinal cord injury: plasticity, regeneration and the challenge of translational drug development. Trends Neurosci. 32, 41-7. 
https://doi.org/10.1016/j.tins.2008.09.008

Bock, P., Beineke, A., Techangamsuwan, S., Baumgärtner, W., Wewetzer, K., 2007. Differential expression of HNK-1 and p75NTR in adult canine Schwann cells and olfactory ensheathing cells in situ but not in vitro. J. Comp. Neurol. 505, 572-585. https://doi.org/10.1002/cne.21519

Bowes, C., Massey, J.M., Burish, M., Cerkevich, C.M., Kaas, J.H., 2012. Chondroitinase ABC promotes selective reactivation of somatosensory cortex in squirrel monkeys after a cervical dorsal column lesion. Proc. Natl. Acad. Sci. U. S. A. 109, 2595-600. https://doi.org/10.1073/pnas.1121604109

Bradbury, E.J., Carter, L.M., 2011. Manipulating the glial scar: Chondroitinase ABC as a therapy for spinal cord injury. Brain Res. Bull. 84, 306-316. https://doi.org/10.1016/j.brainresbull.2010.06.015

Bradbury, E.J., Khemani, S., Von R, K., Priestley, J. V., McMahon, S.B., 1999. NT-3 promotes growth of lesioned adult rat sensory axons ascending in the dorsal columns of the spinal cord. Eur. J. Neurosci. 11, 3873-3883. https://doi.org/10.1046/j.1460-9568.1999.00809.x

Bradbury, E.J., Moon, L.D.F., Popat, R.J., King, V.R., Bennett, G.S., Patel, P.N., Fawcett, J.W., McMahon, S.B., 2002. Chondroitinase ABC promotes functional recovery after spinal cord injury. Nature 416, 636-40. https://doi.org/10.1038/416636a

Bretzner, F., Liu, J., Currie, E., Roskams, A.J., Tetzlaff, W., 2008. Undesired effects of a combinatorial treatment for spinal cord injury - transplantation of olfactory ensheathing cells and BDNF infusion to the red nucleus. Eur. J. Neurosci. 28, 1795-1807. https://doi.org/10.1111/j.14609568.2008.06462.x

Bretzner, F., Plemel, J.R., Liu, J., Richter, M., Roskams, A.J., Tetzlaff, W., 2010. Combination of olfactory ensheathing cells with local versus systemic cAMP treatment after a cervical rubrospinal tract injury. J. Neurosci. Res. 88, n/a-n/a. https://doi.org/10.1002/jnr.22440

Brückner, G., Bringmann, A., Härtig, W., Köppé, G., Delpech, B., Brauer, K., 1998. Acute and longlasting changes in extracellular-matrix chondroitin-sulphate proteoglycans induced by injection of chondroitinase $A B C$ in the adult rat brain. Exp Brain Res 121, 300-310.

Burnside, E.R., De Winter, F., Didangelos, A., James, N.D., Andreica, E.-C., Layard-Horsfall, H., Muir, E.M., Verhaagen, J., Bradbury, E.J., 2018. Immune-evasive gene switch enables regulated delivery of chondroitinase after spinal cord injury. Brain 141, 2362-2381. https://doi.org/10.1093/brain/awy158

Caggiano, A.O., Zimber, M.P., Ganguly, A., Blight, A.R., Gruskin, E.A., 2005. Chondroitinase ABCI Improves Locomotion and Bladder Function following Contusion Injury of the Rat Spinal Cord. J. Neurotrauma 22, 226-239. https://doi.org/10.1089/neu.2005.22.226

Carwardine, D., Prager, J., Neeves, J., Muir, E.M., Uney, J., Granger, N., Wong, L.-F., 2017. Transplantation of canine olfactory ensheathing cells producing chondroitinase $A B C$ promotes 
chondroitin sulphate proteoglycan digestion and axonal sprouting following spinal cord injury. PLoS One 12, e0188967. https://doi.org/10.1371/journal.pone.0188967

Carwardine, D., Wong, L.-F., Fawcett, J.W., Muir, E.M., Granger, N., 2016. Canine olfactory ensheathing cells from the olfactory mucosa can be engineered to produce active chondroitinase ABC. J. Neurol. Sci. 367, 311-8. https://doi.org/10.1016/j.jns.2016.06.011

Cross, S., n.d. GitHub - MIA: Modular, object-based analysis for ImageJ/Fiji [WWW Document]. URL https://github.com/SJCross/MIA (accessed 11.10.19).

Deumens, R., Koopmans, G.C., Honig, W.M.M., Maquet, V., Jérôme, R., Steinbusch, H.W.M., Joosten, E.A.J., 2006. Chronically injured corticospinal axons do not cross large spinal lesion gaps after a multifactorial transplantation strategy using olfactory ensheathing cell/olfactory nerve fibroblast-biomatrix bridges. J. Neurosci. Res. 83, 811-20. https://doi.org/10.1002/jnr.20768

Du Beau, A., Shakya Shrestha, S., Bannatyne, B.A., Jalicy, S.M., Linnen, S., Maxwell, D.J., 2012. Neurotransmitter phenotypes of descending systems in the rat lumbar spinal cord. Neuroscience 227, 67-79. https://doi.org/10.1016/j.neuroscience.2012.09.037

Dumont, R.J., Okonkwo, D.O., Verma, S., Hurlbert, R.J., Boulos, P.T., Ellegala, D.B., Dumont, A.S., 2001. Acute spinal cord injury, part I: pathophysiologic mechanisms. Clin. Neuropharmacol. 24, 254-64.

Feron, F., Perry, C., Cochrane, J., Licina, P., Nowitzke, A., Urquhart, S., Geraghty, T., Mackay-Sim, A., 2005. Autologous olfactory ensheathing cell transplantation in human spinal cord injury. Brain 128, 2951-2960. https://doi.org/10.1093/brain/awh657

Filli, L., Engmann, A.K., Zörner, B., Weinmann, O., Moraitis, T., Gullo, M., Kasper, H., Schneider, R., Schwab, M.E., 2014. Bridging the gap: A reticulo-propriospinal detour bypassing an incomplete spinal cord injury. J. Neurosci. 34, 13399-13410. https://doi.org/10.1523/JNEUROSCI.070114.2014

Fouad, K., Pearse, D.D., Tetzlaff, W., Vavrek, R., 2009. Transplantation and repair: Combined cell implantation and chondroitinase delivery prevents deterioration of bladder function in rats with complete spinal cord injury. Spinal Cord 47, 727-732. https://doi.org/10.1038/sc.2009.10

Fouad, K., Schnell, L., Bunge, M.B., Schwab, M.E., Liebscher, T., Pearse, D.D., 2005. Combining Schwann Cell Bridges and Olfactory-Ensheathing Glia Grafts with Chondroitinase Promotes Locomotor Recovery after Complete Transection of the Spinal Cord. J. Neurosci. 25.

Führmann, T., Tam, R.Y., Ballarin, B., Coles, B., Elliott Donaghue, I., van der Kooy, D., Nagy, A., Tator, C.H., Morshead, C.M., Shoichet, M.S., 2016. Injectable hydrogel promotes early survival of induced pluripotent stem cell-derived oligodendrocytes and attenuates longterm teratoma formation in a spinal cord injury model. Biomaterials 83, 23-36.

https://doi.org/10.1016/j.biomaterials.2015.12.032

García-Alías, G., Barkhuysen, S., Buckle, M., Fawcett, J.W., 2009. Chondroitinase ABC treatment 
opens a window of opportunity for task-specific rehabilitation. Nat. Neurosci. 12, 1145-51. https://doi.org/10.1038/nn.2377

García-Alías, G., Fawcett, J.W., 2012. Training and anti-CSPG combination therapy for spinal cord injury. Exp. Neurol. 235, 26-32. https://doi.org/10.1016/j.expneurol.2011.09.009

García-Alías, G., Lin, R., Akrimi, S.F., Story, D., Bradbury, E.J., Fawcett, J.W., 2008. Therapeutic time window for the application of chondroitinase $A B C$ after spinal cord injury. Exp. Neurol. 210, 331-8. https://doi.org/10.1016/j.expneurol.2007.11.002

Girgis, J., Merrett, D., Kirkland, S., Metz, G.A.S., Verge, V., Fouad, K., 2007. Reaching training in rats with spinal cord injury promotes plasticity and task specific recovery. Brain 130, 2993-3003. https://doi.org/10.1093/brain/awm245

Granger, N., Blamires, H., Franklin, R.J.M., Jeffery, N.D., 2012. Autologous olfactory mucosal cell transplants in clinical spinal cord injury: A randomized double-blinded trial in a canine translational model. Brain 135, 3227-3237. https://doi.org/10.1093/brain/aws268

Granger, N., Franklin, R.J.M., Jeffery, N.D., 2014. Cell Therapy for Spinal Cord Injuries: What Is Really Going on? Neurosci. 20, 623-638. https://doi.org/10.1177/1073858413514635

Greenhalgh, A.D., David, S., Bennett, F.C., 2020. Immune cell regulation of glia during CNS injury and disease. Nat. Rev. Neurosci. https://doi.org/10.1038/s41583-020-0263-9

Grider, M.H., Chen, Q., David Shine, H., 2006. Semi-automated quantification of axonal densities in labeled CNS tissue. J. Neurosci. Methods 155, 172-179.

https://doi.org/10.1016/J.JNEUMETH.2005.12.021

Griffin, J.M., Bradke, F., 2020. Therapeutic repair for spinal cord injury: combinatory approaches to address a multifaceted problem. EMBO Mol. Med. 12, e11505.

https://doi.org/10.15252/emmm.201911505

Guérout, N., Derambure, C., Drouot, L., Bon-Mardion, N., Duclos, C., Boyer, O., Marie, J.P., 2010. Comparative gene expression profiling of olfactory ensheathing cells from olfactory bulb and olfactory mucosa. Glia 58, 1570-1580. https://doi.org/10.1002/glia.21030

Guest, J.D., Herrera, L., Margitich, I., Oliveria, M., Marcillo, A., Casas, C.E., 2008. Xenografts of expanded primate olfactory ensheathing glia support transient behavioral recovery that is independent of serotonergic or corticospinal axonal regeneration in nude rats following spinal cord transection. Exp. Neurol. 212, 261-74. https://doi.org/10.1016/j.expneurol.2008.03.010

Houle, J.D., Tom, V.J., Mayes, D., Wagoner, G., Phillips, N., Silver, J., 2006. Combining an autologous peripheral nervous system "bridge" and matrix modification by chondroitinase allows robust, functional regeneration beyond a hemisection lesion of the adult rat spinal cord. J. Neurosci.

26, 7405-7415. https://doi.org/10.1523/JNEUROSCI.1166-06.2006

Hu, H.Z., Granger, N., Pai, S.B., Bellamkonda, R. V, Jeffery, N.D., 2018. Therapeutic efficacy of microtube-embedded chondroitinase $A B C$ in a canine clinical model of spinal cord injury. Brain 
141, 1017-1027. https://doi.org/10.1093/brain/awy007

Ibrahim, A., Li, D., Collins, A., Tabakow, P., Raisman, G., Li, Y., 2014. Comparison of Olfactory Bulbar and Mucosal Cultures in a Rat Rhizotomy Model. Cell Transplant. 23, 1465-1470. https://doi.org/10.3727/096368913X676213

Imaizumi, T., Lankford, K.L., Waxman, S.G., Greer, C.A., Kocsis, J.D., 1998. Transplanted Olfactory Ensheathing Cells Remyelinate and Enhance Axonal Conduction in the Demyelinated Dorsal Columns of the Rat Spinal Cord. J. Neurosci. 18, 6176-6185.

Ito, D., Carwardine, D., Prager, J., Wong, L.F., Kitagawa, M., Jeffery, N., Granger, N., 2019. Methods of olfactory ensheathing cell harvesting from the olfactory mucosa in dogs. PLoS One 14, e0213252. https://doi.org/10.1371/journal.pone.0213252

Ito, D., Fujita, N., Ibanez, C., Sasaki, N., Franklin, R.J.M., Jeffery, N.D., 2008. Serum-free medium provides a clinically relevant method to increase olfactory ensheathing cell numbers in olfactory mucosa cell culture. Cell Transplant. 16, 1021-7.

James, N.D., Shea, J., Muir, E.M., Verhaagen, J., Schneider, B.L., Bradbury, E.J., 2015. Chondroitinase gene therapy improves upper limb function following cervical contusion injury. Exp. Neurol. 271, 131-5. https://doi.org/10.1016/j.expneurol.2015.05.022

Jefferson, S.C., Tester, N.J., Howland, D.R., 2011. Chondroitinase ABC promotes recovery of adaptive limb movements and enhances axonal growth caudal to a spinal hemisection. J. Neurosci. 31, 5710-20. https://doi.org/10.1523/JNEUROSCI.4459-10.2011

Jeffery, N.D., Lakatos, A., Franklin, R.J.M., 2005. Autologous olfactory glial cell transplantation is reliable and safe in naturally occurring canine spinal cord injury. J. Neurotrauma 22, 1282-93. https://doi.org/10.1089/neu.2005.22.1282

Jeffery, N.D., Smith, P.M., Lakatos, A., Ibanez, C., Ito, D., Franklin, R.J.M., 2006. Clinical canine spinal cord injury provides an opportunity to examine the issues in translating laboratory techniques into practical therapy. Spinal Cord 44, 584-93. https://doi.org/10.1038/sj.sc.3101912

Kanno, H., Pressman, Y., Moody, A., Berg, R., Muir, E.M., Rogers, J.H., Ozawa, H., Itoi, E., Pearse, D.D., Bunge, M.B., 2014. Combination of Engineered Schwann Cell Grafts to Secrete Neurotrophin and Chondroitinase Promotes Axonal Regeneration and Locomotion after Spinal Cord Injury. J. Neurosci. 34.

Karimi-Abdolrezaee, S., Eftekharpour, E., Wang, J., Schut, D., Fehlings, M.G., 2010. Synergistic effects of transplanted adult neural stem/progenitor cells, chondroitinase, and growth factors promote functional repair and plasticity of the chronically injured spinal cord. J. Neurosci. 30, 1657-1676. https://doi.org/10.1523/JNEUROSCI.3111-09.2010

Kawaja, M.D., Boyd, J.G., Smithson, L.J., Jahed, A., Doucette, R., 2009. Technical Strategies to Isolate Olfactory Ensheathing Cells for Intraspinal Implantation. J. Neurotrauma 26, 155-177. https://doi.org/10.1089/neu.2008.0709 
Kennedy, P.R., 1990. Corticospinal, rubrospinal and rubro-olivary projections: a unifying hypothesis. Trends Neurosci. 13, 474-479. https://doi.org/10.1016/0166-2236(90)90079-P

Khankan, R.R., Griffis, K.G., Haggerty-Skeans, J.R., Zhong, H., Roy, R.R., Edgerton, V.R., Phelps, P.E., 2016. Olfactory Ensheathing Cell Transplantation after a Complete Spinal Cord Transection Mediates Neuroprotective and Immunomodulatory Mechanisms to Facilitate Regeneration. J. Neurosci. 36, 6269-86. https://doi.org/10.1523/JNEUROSCI.0085-16.2016

Lakatos, A., Barnett, S.C., Franklin, R.J.M., 2003. Olfactory ensheathing cells induce less host astrocyte response and chondroitin sulphate proteoglycan expression than Schwann cells following transplantation into adult CNS white matter. Exp. Neurol. 184, 237-246. https://doi.org/10.1016/S0014-4886(03)00270-X

Lee, H., McKeon, R.J., Bellamkonda, R. V, 2010. Sustained delivery of thermostabilized chABC enhances axonal sprouting and functional recovery after spinal cord injury. Proc. Natl. Acad. Sci. U. S. A. 107, 3340-5. https://doi.org/10.1073/pnas.0905437106

Li, L., Adnan, H., Xu, B., Wang, J., Wang, C., Li, F., Tang, K., 2015. Effects of transplantation of olfactory ensheathing cells in chronic spinal cord injury: a systematic review and meta-analysis. Eur. Spine J. 24, 919-930. https://doi.org/10.1007/s00586-014-3416-6

Li, Y., Field, P.M., Raisman, G., 1998. Regeneration of adult rat corticospinal axons induced by transplanted olfactory ensheathing cells. J. Neurosci. 18, 10514-24.

Li, Y., Field, P.M., Raisman, G., 1997. Repair of Adult Rat Corticospinal Tract by Transplants of Olfactory Ensheathing Cells. Science (80-. ). 277.

Li, Y., Yu, H.L., Chen, L.F., Duan, C.X., Zhang, J.Y., Li, B.C., 2010. Survival and number of olfactory ensheathing cells transplanted in contused spinal cord of rats. Chinese J. Traumatol. - English Ed. 13, 356-361. https://doi.org/10.3760/cma.j.issn.1008-1275.2010.06.007

Lima, C., Escada, P., Pratas-Vital, J., Branco, C., Arcangeli, C.A., Lazzeri, G., Maia, C.A.S., Capucho, C., Hasse-Ferreira, A., Peduzzi, J.D., 2010. Olfactory mucosal autografts and rehabilitation for chronic traumatic spinal cord injury. Neurorehabil. Neural Repair 24, 10-22. https://doi.org/10.1177/1545968309347685

Lima, C., Pratas-Vital, J., Escada, P., Hasse-Ferreira, A., Capucho, C., Peduzzi, J.D., 2006. Olfactory Mucosa Autografts in Human Spinal Cord Injury: A Pilot Clinical Study. J Spinal Cord Med 29, 191-203.

Lin, R., Kwok, J.C.F., Crespo, D., Fawcett, J.W., 2008. Chondroitinase ABC has a long-lasting effect on chondroitin sulphate glycosaminoglycan content in the injured rat brain. J. Neurochem. 108, 400-408. https://doi.org/10.1111/j.1471-4159.2007.05066.x

Lu, J., Féron, F., Mackay-Sim, A., Waite, P.M.E., 2002. Olfactory ensheathing cells promote locomotor recovery after delayed transplantation into transected spinal cord. Brain 125, 14-21.

Mackay-Sim, A., Féron, F., Cochrane, J., Bassingthwaighte, L., Bayliss, C., Davies, W., Fronek, P., Gray, 
C., Kerr, G., Licina, P., Nowitzke, A., Perry, C., Silburn, P.A.S., Urquhart, S., Geraghty, T., 2008. Autologous olfactory ensheathing cell transplantation in human paraplegia: A 3-year clinical trial. Brain 131, 2376-2386. https://doi.org/10.1093/brain/awn173

Mayeur, A., Duclos, C., Honoré, A., Gauberti, M., Drouot, L., do Rego, J.-C., Bon-Mardion, N., Jean, L., Vérin, E., Emery, E., Lemarchant, S., Vivien, D., Boyer, O., Marie, J.-P., Guérout, N., 2013. Potential of Olfactory Ensheathing Cells from Different Sources for Spinal Cord Repair. PLoS One 8, e62860. https://doi.org/10.1371/journal.pone.0062860

Meijering, E., 2003. FeatureJ [WWW Document]. URL https://imagescience.org/meijering/software/featurej/ (accessed 11.1.19).

Milone, M.C., O’Doherty, U., 2018. Clinical use of lentiviral vectors. Leukemia. https://doi.org/10.1038/s41375-018-0106-0

Montoya, C.P., Campbell-Hope, L.J., Pemberton, K.D., Dunnett, S.B., 1991. The "staircase test": a measure of independent forelimb reaching and grasping abilities in rats. J. Neurosci. Methods 36, 219-228. https://doi.org/10.1016/0165-0270(91)90048-5

Moon, L.D.F., Asher, R.A., Rhodes, K.E., Fawcett, J.W., 2001. Regeneration of CNS axons back to their target following treatment of adult rat brain with chondroitinase ABC. Nat. Neurosci. 4, 465466. https://doi.org/10.1038/87415

Moore, S.A., Granger, N., Olby, N.J., Spitzbarth, I., Jeffery, N.D., Tipold, A., Nout-Lomas, Y.S., da Costa, R.C., Stein, V.M., Noble-Haeusslein, L.J., Blight, A.R., Grossman, R.G., Basso, D.M., Levine, J.M., 2017. Targeting Translational Successes through CANSORT-SCI: Using Pet Dogs To Identify Effective Treatments for Spinal Cord Injury. J. Neurotrauma 34, 2007-2018. https://doi.org/10.1089/neu.2016.4745

Muir, E., De Winter, F., Verhaagen, J., Fawcett, J., 2019. Recent advances in the therapeutic uses of chondroitinase ABC. Exp. Neurol. 113032. https://doi.org/10.1016/j.expneurol.2019.113032

Muir, E.M., Fyfe, I., Gardiner, S., Li, L., Warren, P., Fawcett, J.W., Keynes, R.J., Rogers, J.H., 2010. Modification of $\mathrm{N}$-glycosylation sites allows secretion of bacterial chondroitinase ABC from mammalian cells. J. Biotechnol. 145, 103-110. https://doi.org/10.1016/j.jbiotec.2009.11.002

Nakhjavan-Shahraki, B., Yousefifard, M., Rahimi-Movaghar, V., Baikpour, M., Nasirinezhad, F., Safari, S., Yaseri, M., Moghadas Jafari, A., Ghelichkhani, P., Tafakhori, A., Hosseini, M., 2018.

Transplantation of olfactory ensheathing cells on functional recovery and neuropathic pain after spinal cord injury; systematic review and meta-analysis. Sci. Rep. 8, 325. https://doi.org/10.1038/s41598-017-18754-4

Naldini, L., 2011. Ex vivo gene transfer and correction for cell-based therapies. Nat. Rev. Genet. 12, 301-315. https://doi.org/10.1038/nrg2985

Nash, H.H., Borke, R.C., Anders, J.J., 2002. Ensheathing cells and methylprednisolone promote axonal regeneration and functional recovery in the lesioned adult rat spinal cord. J. Neurosci. 22, 
7111-20. https://doi.org/20026746

Novikova, L.N., Lobov, S., Wiberg, M., Novikov, L.N., 2011. Efficacy of olfactory ensheathing cells to support regeneration after spinal cord injury is influenced by method of culture preparation. Exp. Neurol. 229, 132-142. https://doi.org/10.1016/j.expneurol.2010.09.021

Pastrana, E., Moreno-Flores, M.T., Avila, J., Wandosell, F., Minichiello, L., Diaz-Nido, J., 2007. BDNF production by olfactory ensheathing cells contributes to axonal regeneration of cultured adult CNS neurons. Neurochem. Int. 50, 491-8. https://doi.org/10.1016/j.neuint.2006.10.004

Pearse, D.D., Sanchez, A.R., Pereira, F.C., Andrade, C.M., Puzis, R., Pressman, Y., Golden, K., Kitay, B.M., Blits, B., Wood, P.M., Bunge, M.B., 2007. Transplantaion of Schwann Cells and/or Olfactory Ensheathing Glia into the Contused Spinal Cord: Survival, Migration, Axon Association, and Functional Recovery. Glia 55, 976-1000. https://doi.org/10.1002/glia

Prager, J., Adams, C.F., Delaney, A.M., Chanoit, G., Tarlton, J.F., Wong, L.F., Chari, D.M., Granger, N., 2020. Stiffness-matched biomaterial implants for cell delivery: clinical, intraoperative ultrasound elastography provides a 'target' stiffness for hydrogel synthesis in spinal cord injury. J. Tissue Eng. 11, 1-14. https://doi.org/10.1177/2041731420934806

R Core Team, 2013. R: A language and environment for statistical computing. Found. Stat. Comput. Ramón-Cueto, A., Cordero, M.I., Santos-Benito, F.F., Avila, J., 2000. Functional Recovery of Paraplegic Rats and Motor Axon Regeneration in Their Spinal Cords by Olfactory Ensheathing Glia. Neuron 25, 425-435. https://doi.org/10.1016/S0896-6273(00)80905-8

Ramón-Cueto, A., Plant, G.W., Avila, J., Bunge, M.B., 1998. Long-distance axonal regeneration in the transected adult rat spinal cord is promoted by olfactory ensheathing glia transplants. J. Neurosci. 18, 3803-3815. https://doi.org/10.1523/jneurosci.18-10-03803.1998

Raspa, A., Bolla, E., Cuscona, C., Gelain, F., 2019. Feasible stabilization of chondroitinase abc enables reduced astrogliosis in a chronic model of spinal cord injury. CNS Neurosci. Ther. 25, 86-100. https://doi.org/10.1111/cns.12984

Reshamwala, R., Shah, M., St John, J., Ekberg, J., 2019. Survival and Integration of Transplanted Olfactory Ensheathing Cells are Crucial for Spinal Cord Injury Repair: Insights from the Last 10 Years of Animal Model Studies. Cell Transplant. https://doi.org/10.1177/0963689719883823

Roet, K.C.D., Verhaagen, J., 2014. Understanding the neural repair-promoting properties of olfactory ensheathing cells. Exp. Neurol. 261, 594-609. https://doi.org/10.1016/j.expneurol.2014.05.007

Roloff, F., Ziege, S., Baumgärtner, W., Wewetzer, K., Bicker, G., 2013. Schwann cell-free adult canine olfactory ensheathing cell preparations from olfactory bulb and mucosa display differential migratory and neurite growth-promoting properties in vitro. BMC Neurosci. 14, 141. https://doi.org/10.1186/1471-2202-14-141

Rosenzweig, E.S., Salegio, E.A., Liang, J.J., Weber, J.L., Weinholtz, C.A., Brock, J.H., Moseanko, R., Hawbecker, S., Pender, R., Cruzen, C.L., laci, J.F., Caggiano, A.O., Blight, A.R., Haenzi, B., Huie, 
J.R., Havton, L.A., Nout-Lomas, Y.S., Fawcett, J.W., Ferguson, A.R., Beattie, M.S., Bresnahan, J.C., Tuszynski, M.H., 2019. Chondroitinase improves anatomical and functional outcomes after primate spinal cord injury. Nat. Neurosci. https://doi.org/10.1038/s41593-019-0424-1

Rotterman, T.M., Nardelli, P., Cope, T.C., Alvarez, F.J., 2014. Normal distribution of VGLUT1 synapses on spinal motoneuron dendrites and their reorganization after nerve injury. J. Neurosci. 34, 3475-3492. https://doi.org/10.1523/JNEUROSCI.4768-13.2014

Ruitenberg, M.J., Levison, D.B., Lee, S.V., Verhaagen, J., Harvey, A.R., Plant, G.W., 2005. NT-3 expression from engineered olfactory ensheathing glia promotes spinal sparing and regeneration. Brain 128, 839-853. https://doi.org/10.1093/brain/awh424

Sacrey, L.-A.R., Alaverdashvili, M., Whishaw, I.Q., 2009. Similar hand shaping in reaching-for-food (skilled reaching) in rats and humans provides evidence of homology in release, collection, and manipulation movements. Behav. Brain Res. 204, 153-161. https://doi.org/10.1016/J.BBR.2009.05.035

Sarnat, H.B., Flores-Sarnat, L., 2016. Might the olfactory bulb be an origin of olfactory auras in focal epilepsy? Epileptic Disord. https://doi.org/10.1684/epd.2016.0869

Schindelin, J., Arganda-Carreras, I., Frise, E., Kaynig, V., Longair, M., Pietzsch, T., Preibisch, S., Rueden, C., Saalfeld, S., Schmid, B., Tinevez, J.-Y., White, D.J., Hartenstein, V., Eliceiri, K., Tomancak, P., Cardona, A., 2012. Fiji: an open-source platform for biological-image analysis. Nat. Methods 9, 676-682. https://doi.org/10.1038/nmeth.2019

Silva, N.A., Sousa, N., Reis, R.L., Salgado, A.J., 2014. From basics to clinical: a comprehensive review on spinal cord injury. Prog. Neurobiol. 114, 25-57. https://doi.org/10.1016/j.pneurobio.2013.11.002

Silver, J., Miller, J.H., 2004. Regeneration beyond the glial scar. Nat. Rev. Neurosci. 5, 146-156. https://doi.org/10.1038/nrn1326

Smith, P.M., Lakatos, A., Barnett, S.C., Jeffery, N.D., Franklin, R.J.M., 2002. Cryopreserved cells isolated from the adult canine olfactory bulb are capable of extensive remyelination following transplantation into the adult rat CNS. Exp. Neurol. 176, 402-6.

Steinmetz, M.P., Horn, K.P., Tom, V.J., Miller, J.H., Busch, S.A., Nair, D., Silver, D.J., Silver, J., 2005. Chronic enhancement of the intrinsic growth capacity of sensory neurons combined with the degradation of inhibitory proteoglycans allows functional regeneration of sensory axons through the dorsal root entry zone in the mammalian spinal cord. J. Neurosci. 25, 8066-8076. https://doi.org/10.1523/JNEUROSCI.2111-05.2005

Steward, O., Sharp, K., Selvan, G., Hadden, A., Hofstadter, M., Au, E., Roskams, J., 2006. A reassessment of the consequences of delayed transplantation of olfactory lamina propria following complete spinal cord transection in rats. Exp. Neurol. 198, 483-99. https://doi.org/10.1016/j.expneurol.2005.12.034 
Tabakow, P., Jarmundowicz, W., Czapiga, B., Fortuna, W., Miedzybrodzki, R., Czyz, M., Huber, J., Szarek, D., Okurowski, S., Szewczyk, P., Gorski, A., Raisman, G., 2013. Transplantation of autologous olfactory ensheathing cells in complete human spinal cord injury. Cell Transplant. 22, 1591-612. https://doi.org/10.3727/096368912X663532

Takeoka, A., Jindrich, D.L., Muñoz-Quiles, C., Zhong, H., van den Brand, R., Pham, D.L., Ziegler, M.D., Ramón-Cueto, A., Roy, R.R., Edgerton, V.R., Phelps, P.E., 2011. Axon Regeneration Can Facilitate or Suppress Hindlimb Function after Olfactory Ensheathing Glia Transplantation. J. Neurosci. 31.

Tester, N.J., Howland, D.R., 2008. Chondroitinase ABC improves basic and skilled locomotion in spinal cord injured cats. Exp. Neurol. 209, 483-496. https://doi.org/10.1016/j.expneurol.2007.07.019

Tester, N.J., Plaas, A.H., Howland, D.R., 2007. Effect of body temperature on chondroitinase ABC's ability to cleave chondroitin sulfate glycosaminoglycans. J. Neurosci. Res. 85, 1110-1118. https://doi.org/10.1002/jnr.21199

Tetzlaff, W., Fouad, K., Kwon, B., 2009. Be careful what you train for. Nat. Neurosci. 12, 1077-1079. https://doi.org/10.1038/nn0909-1077

Todd, A.J., Hughes, D.I., Polgár, E., Nagy, G.G., Mackie, M., Ottersen, O.P., Maxwell, D.J., 2003. The expression of vesicular glutamate transporters VGLUT1 and VGLUT2 in neurochemically defined axonal populations in the rat spinal cord with emphasis on the dorsal horn. Eur. J. Neurosci. 17, 13-27. https://doi.org/10.1046/j.1460-9568.2003.02406.x

Tuszynski, M.H., Steward, O., 2012. Concepts and methods for the study of axonal regeneration in the CNS. Neuron 74, 777-91. https://doi.org/10.1016/j.neuron.2012.05.006

Ulrich, R., Imbschweiler, I., Kalkuhl, A., Lehmbecker, A., Ziege, S., Kegler, K., Becker, K., Deschl, U., Wewetzer, K., Baumgärtner, W., 2014. Transcriptional profiling predicts overwhelming homology of schwann cells, olfactory ensheathing cells, and schwann cell-like glia. Glia 62, 1559-1581. https://doi.org/10.1002/glia.22700

Wang, D., Ichiyama, R.M., Zhao, R., Andrews, M.R., Fawcett, J.W., 2011. Chondroitinase combined with rehabilitation promotes recovery of forelimb function in rats with chronic spinal cord injury. J. Neurosci. 31, 9332-44. https://doi.org/10.1523/JNEUROSCI.0983-11.2011

Warren, P.M., Andrews, M.R., Smith, M., Bartus, K., Bradbury, E.J., Verhaagen, J., Fawcett, J.W., Kwok, J.C.F., 2020. Secretion of a mammalian chondroitinase $A B C$ aids glial integration at PNS/CNS boundaries. Sci. Rep. 10, 1-17. https://doi.org/10.1038/s41598-020-67526-0

Warren, P.M., Steiger, S.C., Dick, T.E., MacFarlane, P.M., Alilain, W.J., Silver, J., 2018. Rapid and robust restoration of breathing long after spinal cord injury. Nat. Commun. 9. https://doi.org/10.1038/s41467-018-06937-0

Watzlawick, R., Rind, J., Sena, E.S., Brommer, B., Zhang, T., Kopp, M.A., Dirnagl, U., Macleod, M.R., 
Howells, D.W., Schwab, J.M., 2016. Olfactory Ensheathing Cell Transplantation in Experimental Spinal Cord Injury: Effect size and Reporting Bias of 62 Experimental Treatments: A Systematic Review and Meta-Analysis. PLOS Biol. 14, e1002468.

https://doi.org/10.1371/journal.pbio.1002468

Welniarz, Q., Dusart, I., Roze, E., 2017. The corticospinal tract: Evolution, development, and human disorders. Dev. Neurobiol. https://doi.org/10.1002/dneu.22455

Whishaw, I.Q., Pellis, S.M., Gorny, B., Kolb, B., Tetzlaff, W., 1993. Proximal and distal impairments in rat forelimb use in reaching follow unilateral pyramidal tract lesions. Behav. Brain Res. 56, 5976. https://doi.org/10.1016/0166-4328(93)90022-I

Zhang, J., Liu, Z., Chen, H., Duan, Z., Zhang, L., Chen, L., Li, B., 2015. Synergic Effects of EPI-NCSCs and OECs on the Donor Cells Migration, the Expression of Neurotrophic Factors, and Locomotor Recovery of Contused Spinal Cord of Rats. J. Mol. Neurosci. 55, 760-769. https://doi.org/10.1007/s12031-014-0416-2

Zhao, R.-R., Fawcett, J.W., 2013. Combination treatment with chondroitinase ABC in spinal cord injury-breaking the barrier. Neurosci. Bull. 29, 477-483. https://doi.org/10.1007/s12264-013$1359-2$ 


\section{Figure Legends}

\section{Figure 1. COEC characterisation prior to transplant}

Canine OEC population was characterised before transplant. An example immunofluorescent image (A) illustrates all cells expressed (p75+, red) and the proportion which have been transduced (GFP+, green). There was no significant difference in the number of transduced GFP+ cells between COECchABC and COEC-GFP (B). Secretion of active chABC by COEC-chABC was confirmed prior to transplant by Morgan Elson reaction ( $C, n=4)$. chABC activity from COEC-GFP was negligible, with a $95 \%$ confidence-interval including 0 . [Mean \pm SD]

\section{Figure 2. COEC quantification and CSPG digestion}

An example immunofluorescent image from a dorsal section of spinal cord shows a group of COECGFPs at 2 weeks after transplant, along with a higher magnification image (A). There was no significant difference between COEC-GFP and COEC-chABC survival (unpaired t-test) (B). Example images of dorsal sections of spinal cord, and higher magnification images (red boxes) show digestion of CSPG (Neurocan, black) and digested stubs of CSPG (C4S, black) around the lesion (marked ${ }^{\dagger}$ ) at 2 weeks after transplant (C). No evidence of CSPG digestion is seen in COEC-GFP group. [Mean \pm SD]

\section{Figure 3. Effect of COECs and COEC-chABC on forepaw reaching recovery}

No significant difference in number of pellets retrieved on Montoya Staircase (A) was seen between transplant groups (COEC-chABC, orange, $n=9$; COEC-GFP, green, $n=11$; media, purple, $n=9$ ), but uninjured sham animals (black, $n=9$ ) did not deteriorate after surgery and were significantly different from all transplanted groups $[F(3,34)=9.67, p<0.0001$ mixed effects analysis, post-hoc Tukey tests as marked]. COEC-ChABC transplanted animals were the only transplant group to significantly increase pellet retrieval after week 1 ( $p=0.036$ post-hoc Tukey test at week 6 peak). Individual number of pellets retrieved and improvement for each animal is shown at week 1 and 6 (B). Accuracy of pellet retrieval on Montoya Staircase (C) showed no significant difference between transplant groups, but a significant difference was seen between all transplant groups and uninjured sham animals $[F(3,34)=5.4, p<0.0038$ mixed effects analysis, post-hoc Tukey test $p<0.0001$ all groups]. Untrained Whishaw reaching at 6 weeks after transplant showed a significantly higher accuracy of retrieval in COEC-chABC transplanted animals compared to both cOEC-GFP and media transplanted animals, similar to sham animals $[F(3,19)=14.8, p<0.0001$ one-way ANOVA, Tukey's post-hoc as marked] (D). Not all animals completed this task (COEC-chABC, $n=4$; cOEC-GFP, $n=5$; media, $n=6$; sham, $n=8$ ).

[Montoya Staircase (A,C) Mean \pm SEM, Whishaw Reaching (D) Mean $\pm S D .{ }^{*}=p<0.05, * *=p<0.01$, $* * * p<0.001, * * * * p<0.0001]$ 


\section{Figure 4. Effect of $C O E C$ and $C O E C-c h A B C$ on ladder crossing and gait}

There was no significant difference in horizontal ladder forelimb foot slips between transplant groups (A), but sham animals were significantly different to all other groups and showed virtually no foot slips $[F(3,32)=28.1, p<0.0001$ mixed effects analysis, post hoc Tukey tests $p<0.0001$ all groups]. Example forelimb gait analysis traces for media and COEC-chABC transplanted animals are shown (B) illustrating stride length (i) and base of support (ii) measures. Both $\operatorname{COEC}-\mathrm{ch} A B C$ and sham animals showed significantly longer fore-limb stride length than media transplanted animals $(C)[F(3,33)=4.9, p=0.0062$ one-way ANOVA, Tukey's post-hoc]. COEC-chABC transplanted animals showed a significantly narrower fore-limb base of support than COEC-GFP and media transplanted animals (D) which was not significantly different to sham animals $[F(3,33)=4.1, p=0.015$, Tukey's post-hoc]. No difference was seen in paw print width between groups $(\mathrm{E})$.

$[$ Mean $\pm S D] *=p<0.05, * *=p<0.01, * * * p<0.001, * * * * p<0.0001]$

\section{Figure 5. BDA axon tracing of corticospinal tract}

BDA labelled CST axons in spinal cord dorsal sections through the lesion $(\dagger)$ for media, COEC-GFP and COEC-chABC transplants are shown (A). White dashed lines show regions of interest for cranial white (i) and grey (ii) matter and caudal white matter (iii). Corresponding higher magnification inset images (red boxes) show magnified white/grey matter border (yellow dashed line, grey matter top) illustrating axon sprouting. Quantified, there is significantly increased BDA positive CST sprouting into cranial grey matter in the COEC-ChABC transplanted group compared to both media and OEC-GFP transplants $(B)[F(2,25)=12.9, p=0.0001$ one-way ANOVA, Tukey's post-hoc]. No significant difference was seen in BDA staining caudal to the lesion (C).

[Mean \pm SD, $* p<0.05, * * p<0.01, * * * p<0.001]$

\section{Figure 6. Serotonergic (5HT) immunostaining caudal to lesion}

Example 5HT immunostained transverse sections $10 \mathrm{~mm}$ caudal to the lesion epicentre are shown for each group (A). Quantification of $5 \mathrm{HT}$ positive staining by automated reference to a pre-specified template (B) shows consistent increased staining in OEC-chABC transplanted group across regions of interest (C-F) [Kruskal-Wallis with post-hoc Dunn's test]. White lower-case letters on template correspond to upper-case figure panels; lateral funiculi (c/C), dorsal grey matter (d/D), ventral grey matter (e/E) and ventral funiculi (f/F).

[Mean $\pm S D, * p<0.05, * * p<0.01, * * * p<0.001]$ 


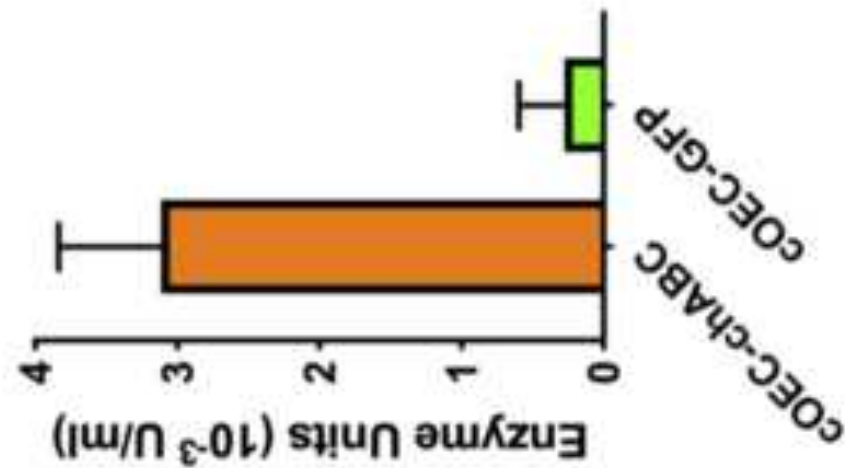

$\cup$
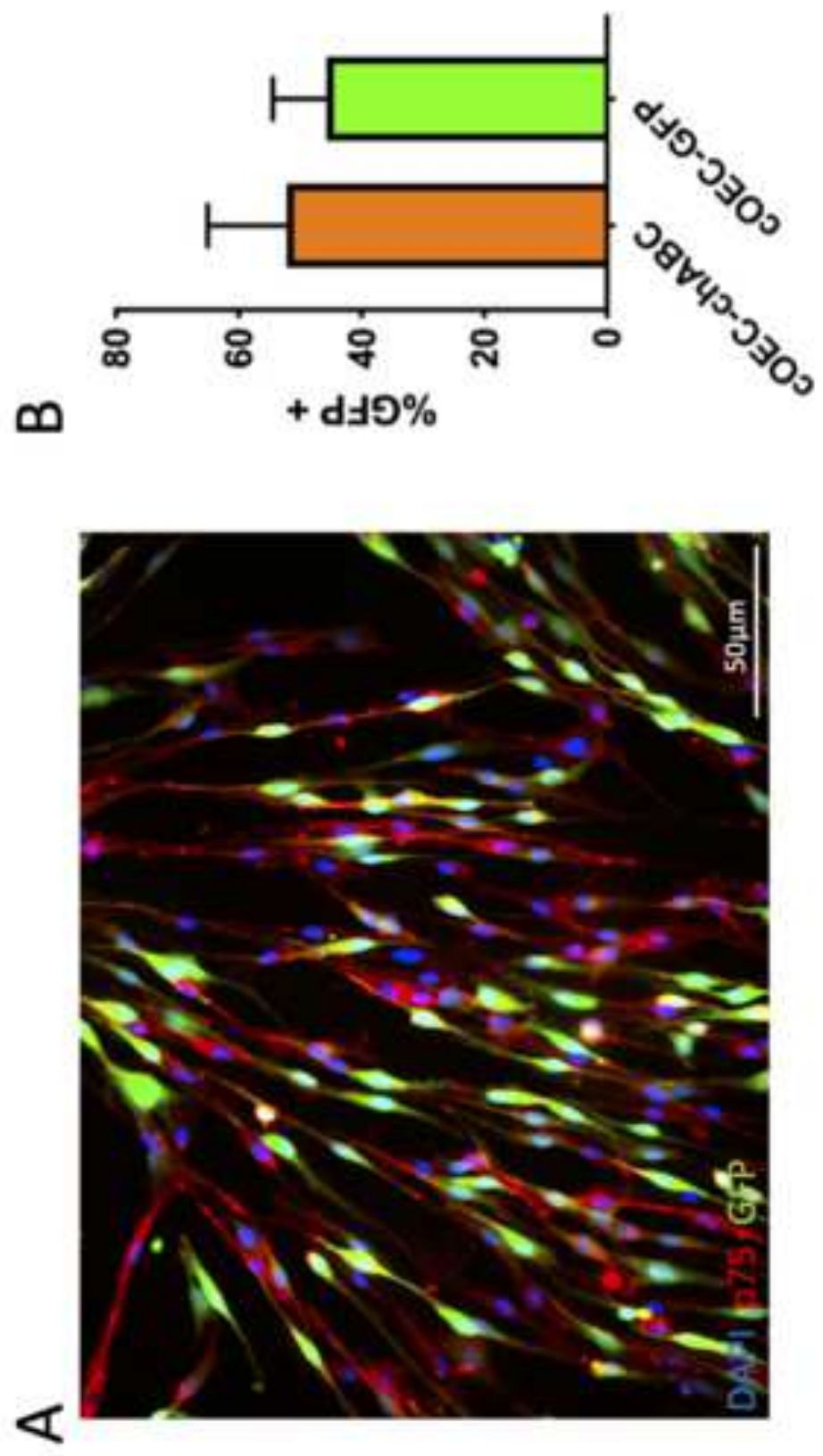
A

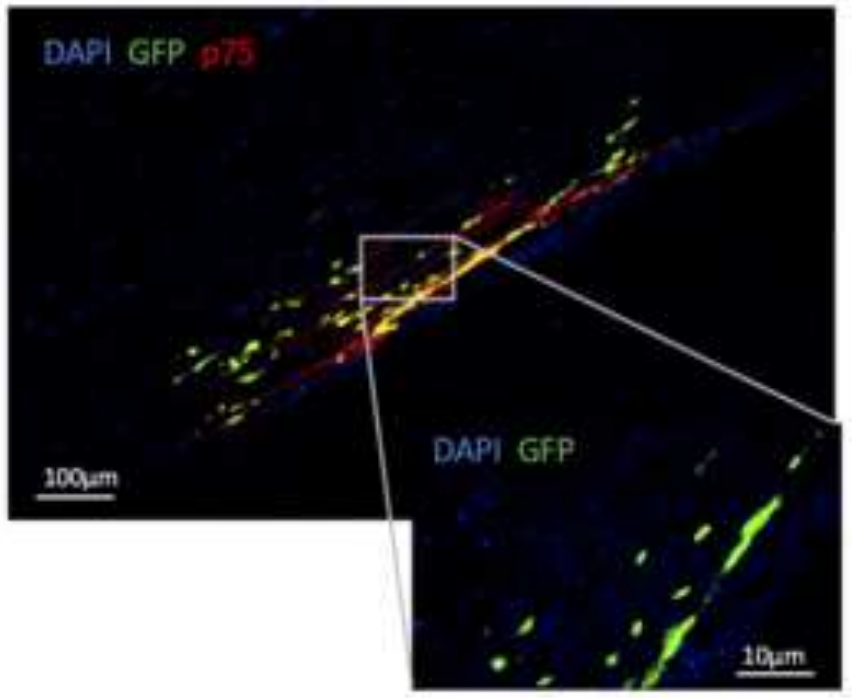

B

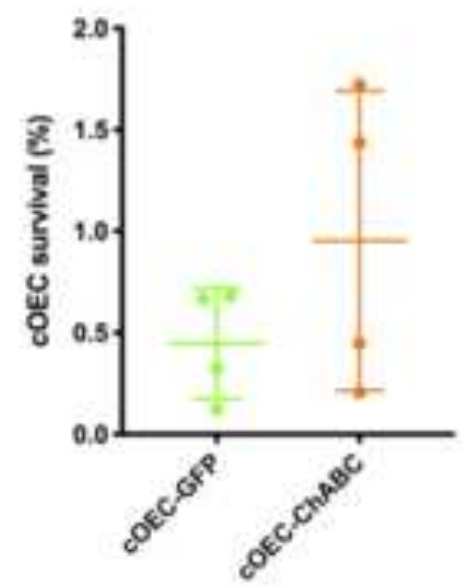

C

\section{CSPG digestion at 2 weeks}

COEC-chABC | Neurocan

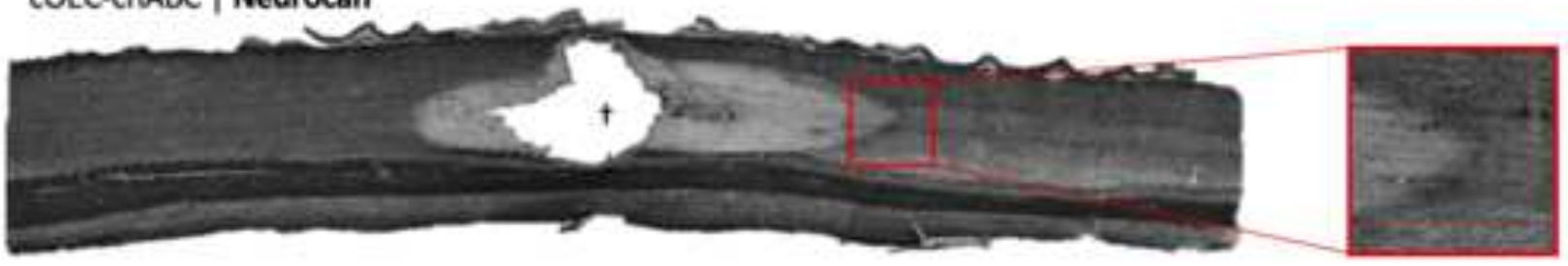

COEC-ChABC | CAS

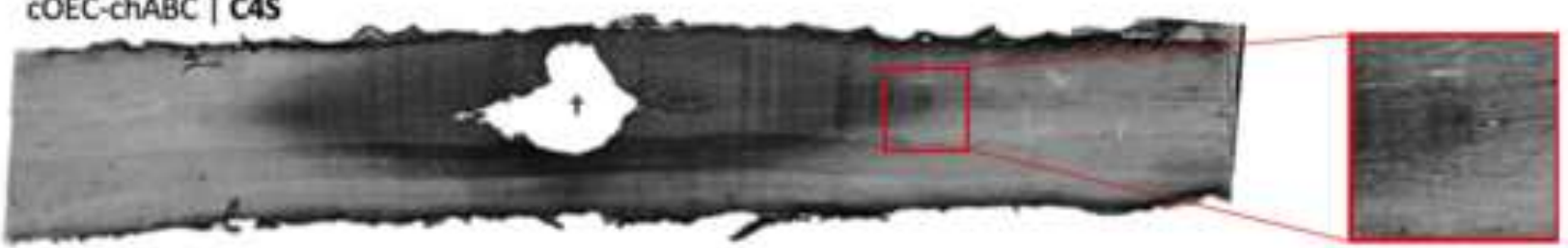

COEC-GFP $\mid$ CAS

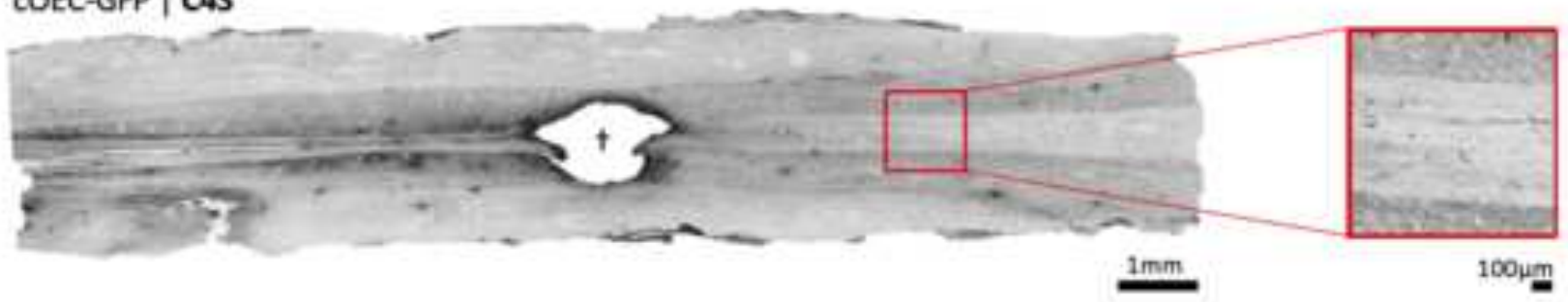




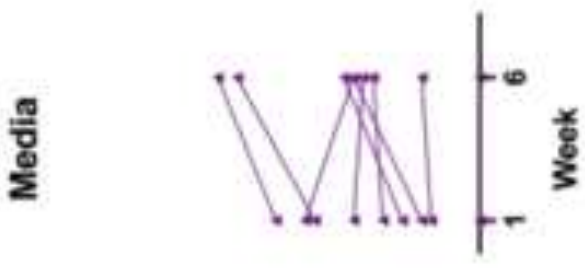

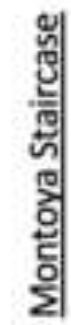

$\infty$
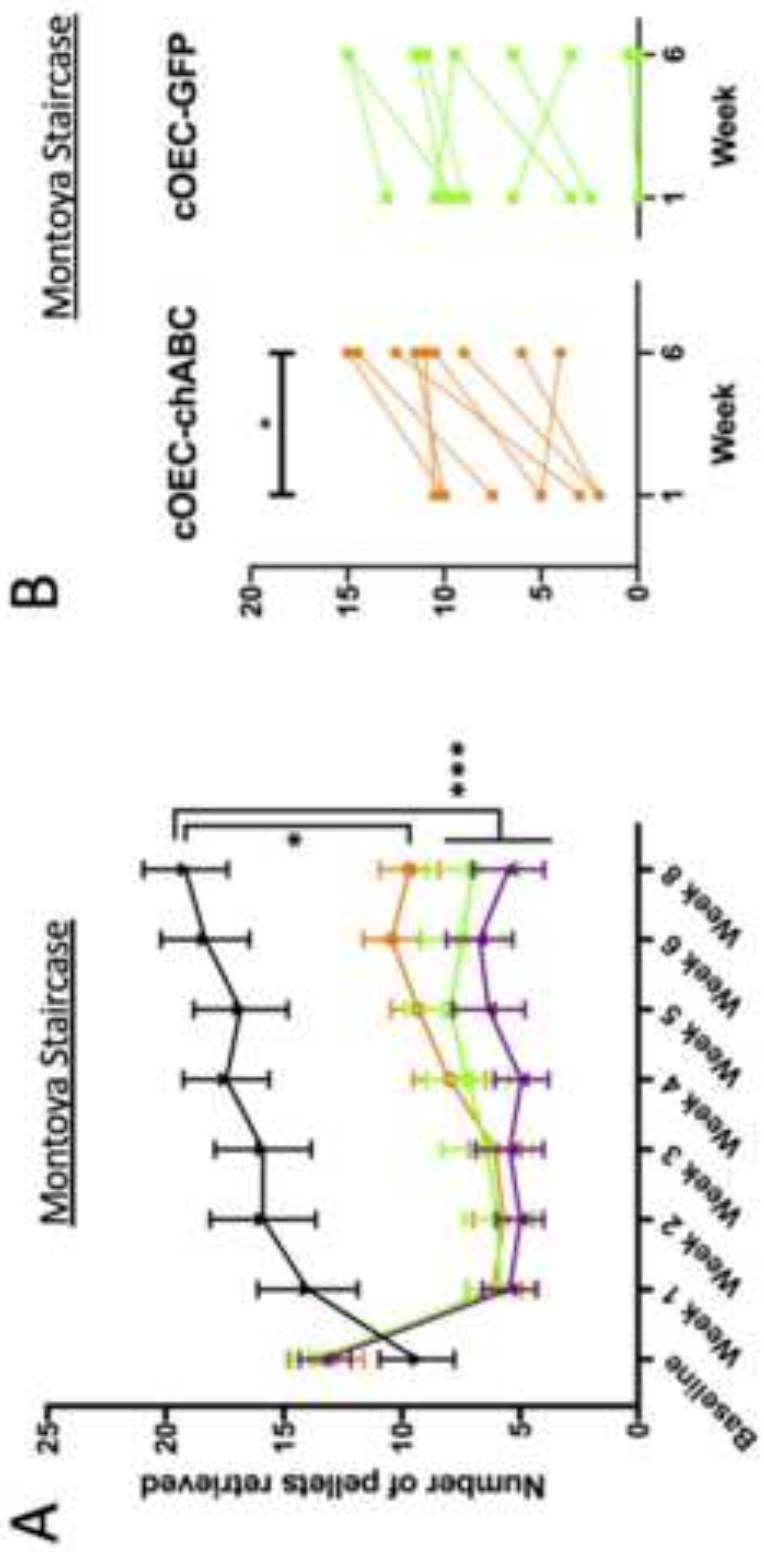

造

岁岁要 $\frac{E}{2}$

$+1+1$

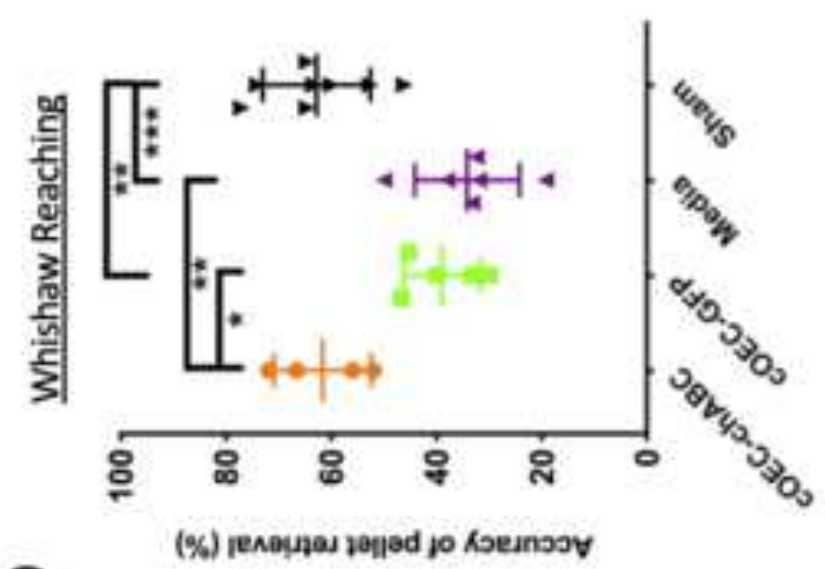

○

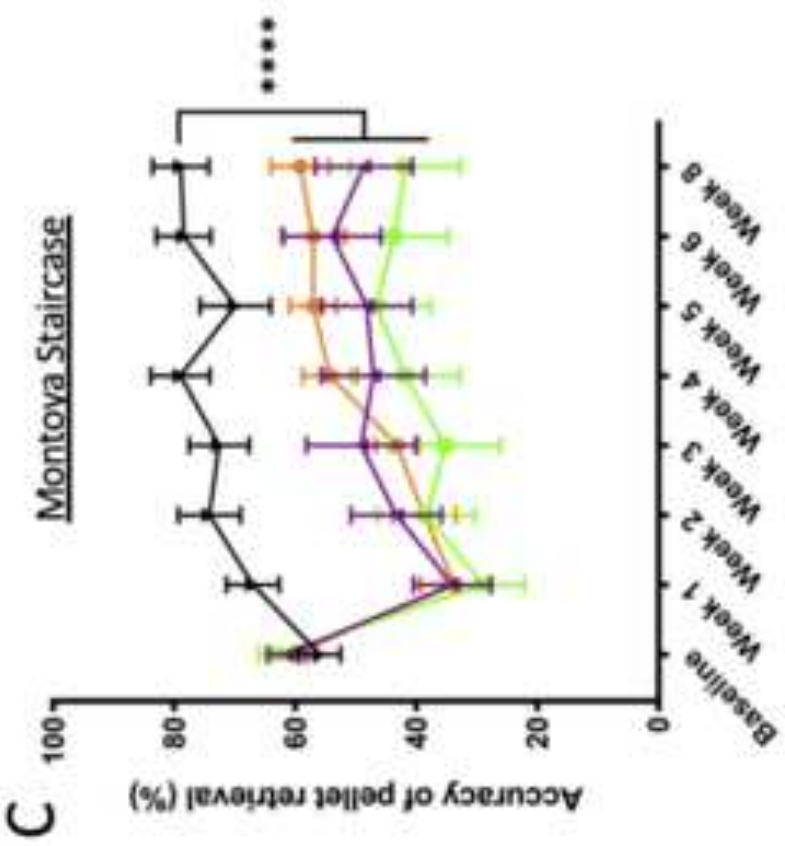


总 $\frac{1}{0}$

岁岁 $\frac{\text { क ल }}{2}$

$+4+1$

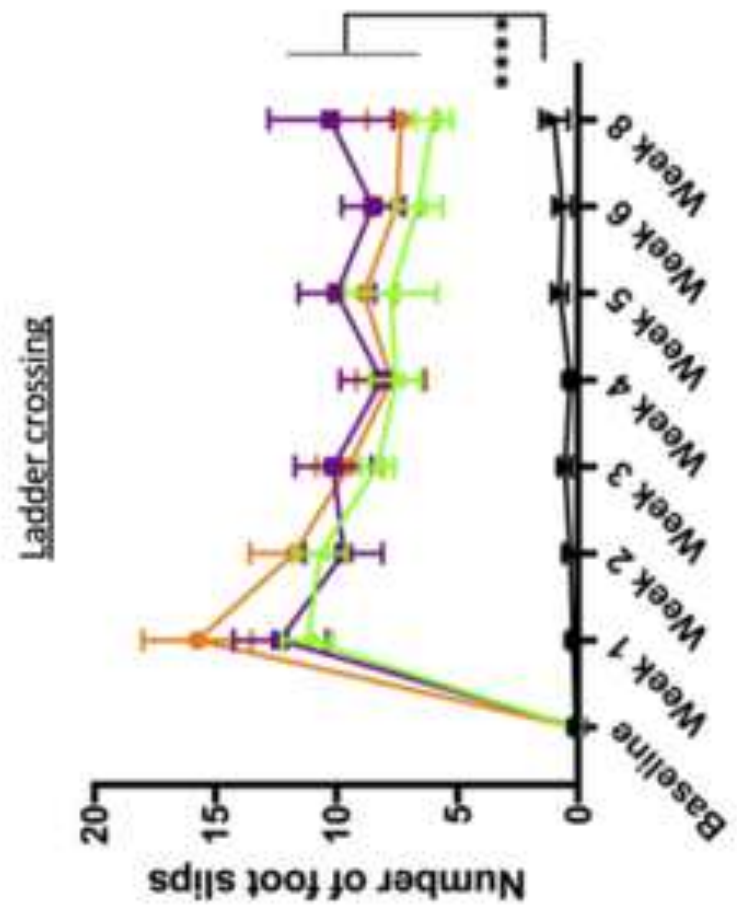

$<$
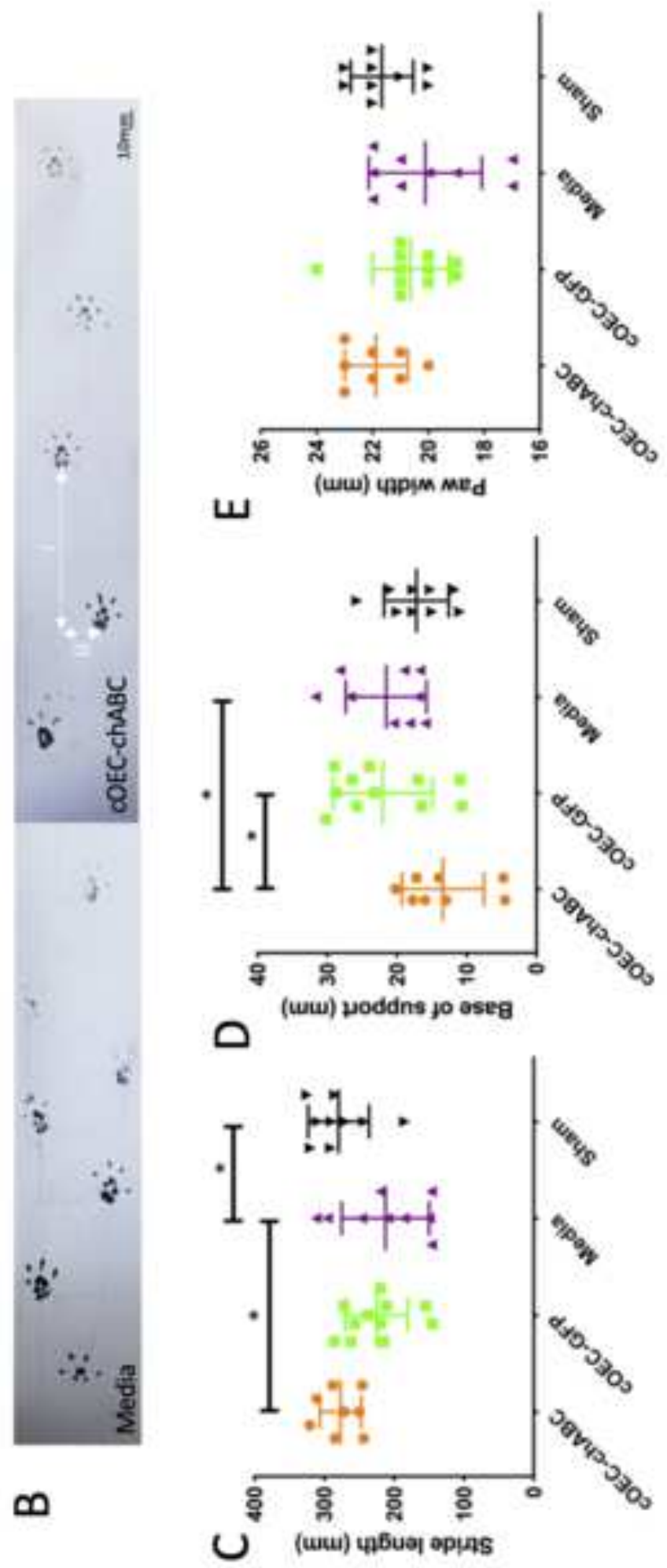

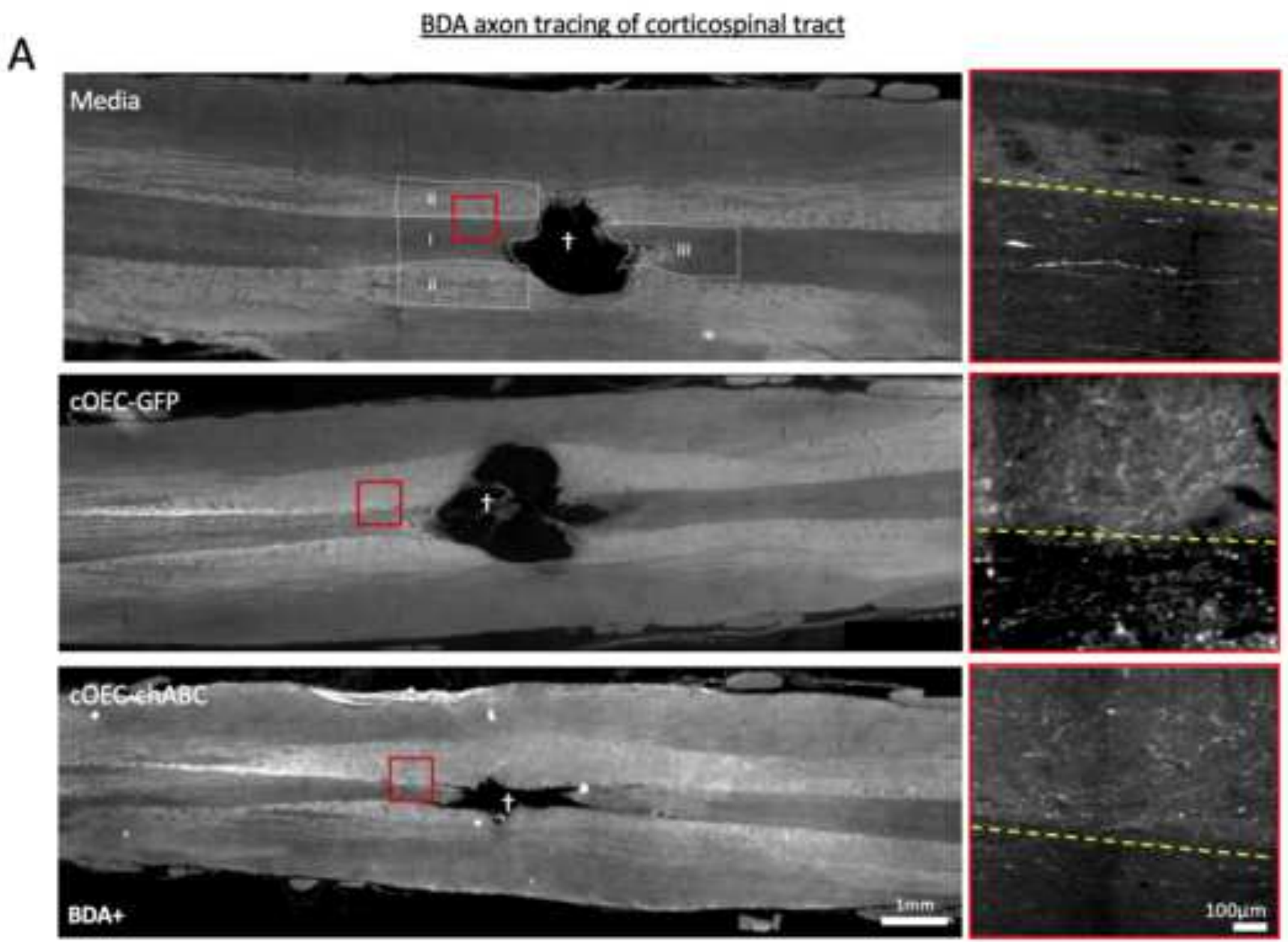

B

CST sprouting into grey matter

C CST axons caudal to lesion
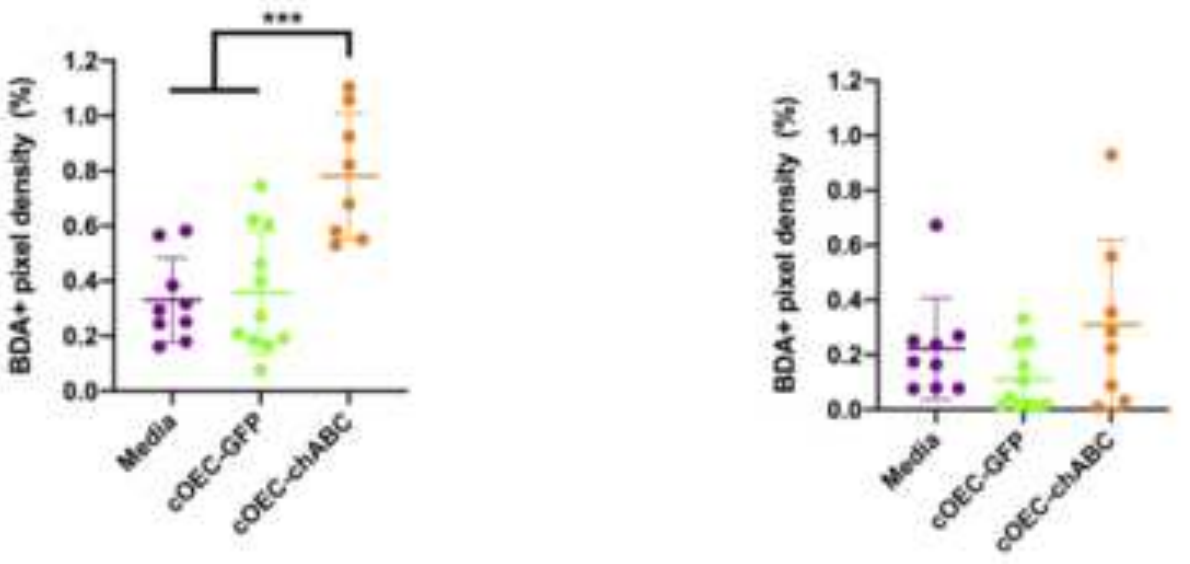


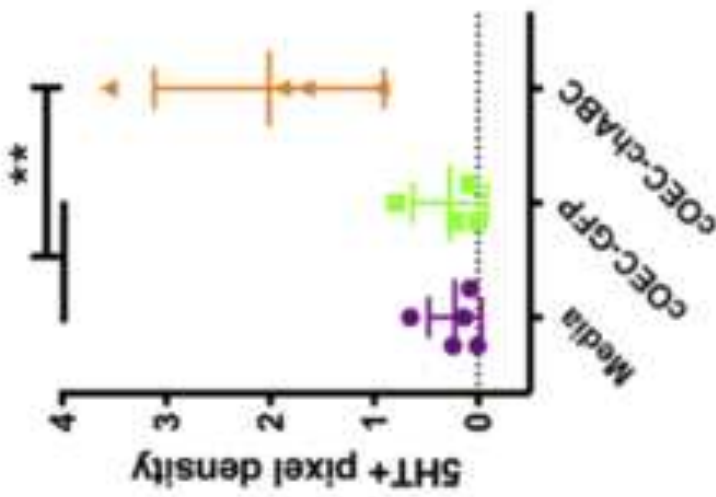

อ

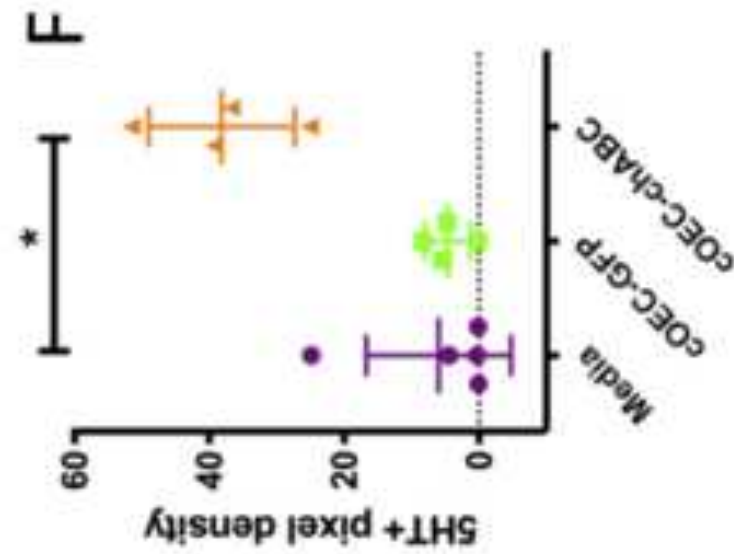

แ4
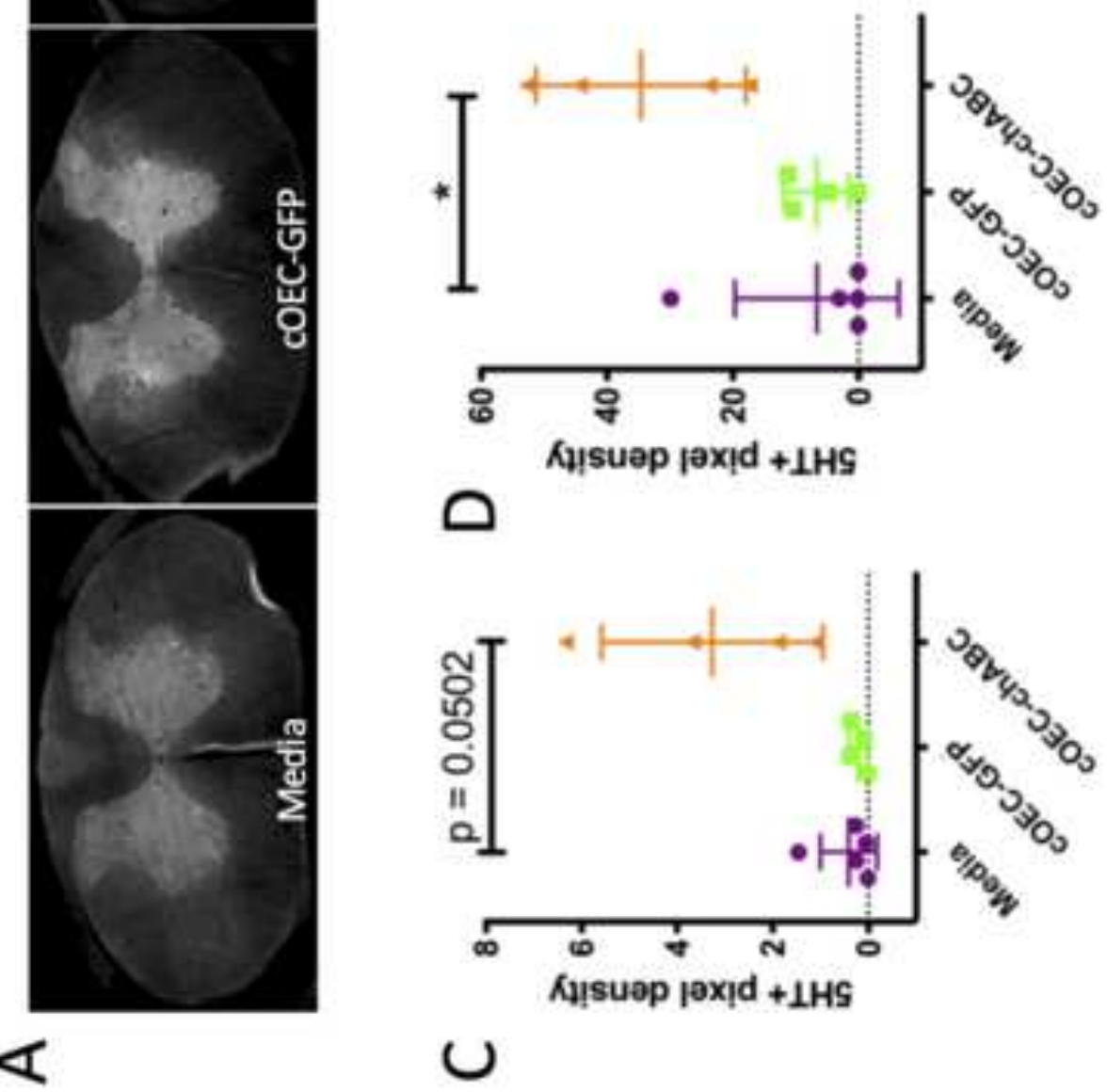

0
0
$\frac{1}{3}$
믄 